\title{
Communication
}

\section{Interplay between Phenotypic Resistance to Relevant Antibiotics in Gram-Negative Urinary Pathogens: A Data-Driven Analysis of 10 Years' Worth of Antibiogram Data}

\author{
Márió Gajdács ${ }^{1, *},+\left[\right.$, Zoltán Bátori ${ }^{2,+}$ and Katalin Burián ${ }^{3}$ (D) \\ 1 Department of Oral Biology and Experimental Dental Research, Faculty of Dentistry, University of Szeged, \\ Tisza Lajos krt. 63., 6720 Szeged, Hungary \\ 2 Department of Ecology, Faculty of Sciences, University of Szeged, Közép fasor 52., 6726 Szeged, Hungary; \\ zbatory@gmail.com \\ 3 Department of Medical Microbiology, Albert Szent-Györgyi Health Center, Faculty of Medicine, University of \\ Szeged, Semmelweis utca 6., 6725 Szeged, Hungary; burian.katalin@med.u-szeged.hu \\ * Correspondence: mariopharma92@gmail.com or gajdacs.mario@stoma.szote.u-szeged.hu; \\ Tel.: +36-62-342-532 \\ + These authors contributed equally to this work.
}

check for

updates

Citation: Gajdács, M.; Bátori, Z.; Burián, K. Interplay between Phenotypic Resistance to Relevant Antibiotics in Gram-Negative Urinary Pathogens: A Data-Driven Analysis of 10 Years' Worth of Antibiogram Data. Life 2021, 11, 1059. https:/ / doi.org/10.3390/life11101059

Academic Editors: Josep M. Sierra and Katalin Prokai-Tatrai

Received: 10 August 2021

Accepted: 5 October 2021

Published: 8 October 2021

Publisher's Note: MDPI stays neutral with regard to jurisdictional claims in published maps and institutional affiliations.

\section{Copyright: (c) 2021 by the authors.} Licensee MDPI, Basel, Switzerland. This article is an open access article distributed under the terms and conditions of the Creative Commons Attribution (CC BY) license (https:// creativecommons.org/licenses/by/ $4.0 /)$.

\begin{abstract}
The global emergence of antimicrobial resistance (AMR) has become a critical issue for clinicians, as it puts the decades of developments in the medical field in jeopardy, by severely limiting the useful therapeutic arsenal of drugs, both in nosocomial and community-acquired infections. In the present study, a secondary analysis of taxonomic and resistance data was performed, corresponding to urinary tract infections (UTIs) caused by Gram-negative bacteria, detected between 1 January 2008 to 31 December 2017 at the Albert Szent-Györgyi Health Center, University of Szeged. The following were identifiable from the data collected: year of isolation; outpatient (OP)/inpatient (IP) origin of the isolate; taxonomy; and susceptibility/resistance to selected indicator antibiotics. Principal component analysis (PCA) and a correlation matrix were used to determine the association between the presences of resistance against indicator antibiotics in each taxonomic group. Overall, data from $n=16,240$ outpatient and $n=13,964$ inpatient Gram-negative UTI isolates were included in the data analyses. In E. coli, strong positive correlations were seen between resistance to ciprofloxacin $(\mathrm{CIP})$ and gentamicin (GEN) resistance (OP: $\mathrm{r}=0.6342, p=0.049$; IP: $\mathrm{r}=0.9602, p<0.001$ ), whereas strong negative correlations were shown for fosfomycin (FOS) and nitrofurantoin (NIT) resistance (OP: $\mathrm{r}=-0.7183, p=0.019$; IP: $\mathrm{r}=-0.7437 ; p=0.014$ ). For Klebsiella spp. isolates, CIP resistance showed strong positive correlation with resistance to third-generation cephalosporins (3GC) and GEN ( $r=0.7976, p=0.006$ and $r=0.7428, p=0.014$, respectively) in OP isolates, and with resistance to trimethoprim-sulfamethoxazole (SXT) and FOS ( $\mathrm{r}=0.8144, p=0.004$ and $\mathrm{r}=0.7758, p<0.001$, respectively) in IP isolates. For members of the Citrobacter-Enterobacter-Serratia group, the resistance among indicator antibiotics showed a strong positive correlation, with the exception of FOS resistance. In the Proteus-Providencia-Morganella group, the strongest association was noted between CIP and SXT resistance (OP: $r=0.9251, p<0.001$; IP: $r=0.8007 ; p=0.005$ ). In the case of OP Acinetobacter spp., CIP showed strong and significant positive correlations with most indicator antibiotics, whereas for IP isolates, strong negative correlations arose among imipenem (IMI) resistance and resistance to other drugs. For Pseudomonas spp., strong and positive correlations were noted among resistance to $\beta$-lactam antibiotics and aminoglycosides, with the exception of ceftazidime (CEFT), showing strong, but negative correlations. Though molecular tests and sequencing-based platforms are now considered as the gold-standard for AMR surveillance, standardized collection of phenotypic resistance data and the introduction of Big Data analytic methods may be a viable alternative for molecular surveillance, especially in low-resource settings.
\end{abstract}

Keywords: urinary tract infection; Gram-negative; antimicrobial drug resistance; MDR; surveillance; big data; principal component analysis; correlation matrix 


\section{Introduction}

The introduction of antibiotics has ushered in a new age of modern medicine, allowing for the successful treatment of previously life-threatening infections, and for the development of many complex medical fields and specialties [1,2]. The global emergence of multidrug resistant (MDR) bacteria has become a critical issue for clinicians, as they put the decades of developments in the medical field in jeopardy, by severely limiting the useful therapeutic arsenal of drugs, both in nosocomial and community-acquired infections [3,4]. The clinical problem of MDR is multifaceted, including the easy access to, and often inappropriate use of, existing antibiotics (i.e., prescriptions in inappropriate indications or for inappropriate time periods, self-medication without prescription), and the lack of development and market authorization of novel antimicrobials $[5,6]$. Warnings have been put out for national governments by both the US and European Centers for Disease Control and Prevention (CDC, ECDC) and the World Health Organization (WHO), calling for global and intersectoral action to address these concerns [7-9]. In fact, the O'Neill report has estimated that by 2050, infectious diseases may lead to the death of 10 million individuals, once again becoming the second leading cause of mortality [10].

Bacteria may become resistant to antibiotics through a variety of mechanisms, and some of these mechanisms are intrinsic, i.e., they are characteristic for the genus/species in question, and resistance is passed on vertically to the daughter cells [11]. Far more commonly, resistance determinants may be found on mobile genetic elements (MGEs), including plasmids, transposons, and integrons [12]. From a public health perspective, these MGEs are especially concerning, as they may carry resistance genes for multiple antibiotics, allowing for the rapid dissemination and outbreak-formation in taxonomicallydiverse bacteria [13]. Resistance-determinants encoded on plasmids have been critical in the development of the MDR phenotype in many relevant pathogens [14]. Additionally, adaptive mechanisms of resistance-including biofilm-formation, metabolic switching, and small-colony variant (SCV) formation - must also be mentioned, most often leading to phenotypic resistance in vivo $[15,16]$. Though methicillin-resistant Staphylococcus aureus (MRSA) may be considered as the first MDR "superbug", after the 2000s, a pronounced shift has been observed towards the relevance of MDR Gram-negative bacteria [15]. This is due to the emergence isolates presenting with extended-spectrum $\beta$-lactamases (ESBLs), carbapenem-resistance (either due to carbapenemases or membrane impermeability) and even colistin-resistance, and the lack of appropriate antimicrobials to treat these infections $[17,18]$.

Urinary tract infections (UTIs) are the second most common infections in developed countries, leading to $7-8$ million visits to primary care physicians and over 1 million visits to the Emergency Department in the US alone $[19,20]$. UTIs also account for substantial economic costs ( $\sim 3-5$ billion US dollars) worldwide, associated with treatment, hospital costs, and productivity losses [21]. Hospitalization rates due to UTIs have also shown an increasing trend worldwide [22]. Although females of a reproductive age are the most commonly burdened with uncomplicated UTIs, the disease may affect anyone, irrespective of age, gender, or socio-economic status [23,24]. The most common causative agent in in both community- and hospital-associated UTIs is Escherichia coli, whereas Gram-negative bacteria are responsible for $80-90 \%$ of all UTIs overall [25]. In older patients, or patients with immunosuppression or other underlying conditions, some species, such as Proteus spp. or non-fermenters (Pseudomonas spp., Acinetobacter spp.), are more commonly found as pathogens [26].

Resistance rates in various geographical regions may show pronounced variations, which should be monitored over time [27]. Continuous surveillance of resistance rates is of great importance, and this data may also be used to aid the introduction and evaluation of antimicrobial stewardship interventions in healthcare institutions [28]. With the advent of molecular methods, next-generation sequencing (NGS), and in-depth bioinformatics analyses in public health microbiology, it has been encouraged that the occurrence and co-occurrence of resistance in clinical isolates be determined on a genetic level, with the 
aim of identifying the most commonly occurring gene(s) responsible for the resistant phenotype, and to ascertain the attributes of successful MDR clones [29]. This data also sheds light on co-occurring resistance mechanisms in Gram-negative bacteria, which may be the basis of driving therapeutic choices in outpatient empirical treatment and the ambulatory use of antibiotics, to avoid the selection of highly-resistant variants [30]. Nevertheless, these methods are costly, time-consuming, not compatible with a large number of clinical isolates, and often only available in national reference laboratories. Therefore, in many clinical microbiology laboratories, reporting of resistance rates (for epidemiological purposes) from clinical isolates heavily relies on phenotypic antimicrobial susceptibility results and tests based on biochemical reactions (e.g., for carbapenemasedetection) [31]. Still, systematically-collected phenotypic resistance data over a long time period may also provide important insights on the association of resistance with clinically important antibiotic groups [32].

In our previous report, we sought to describe the epidemiological aspects and resistance rates of Gram-negative pathogens-including members of the Enterobacterales order and non-fermenters-implicated in UTIs from inpatients and outpatients in a large tertiary-care hospital in Southern Hungary. In the article, the levels of UDR (usual drug resistance), MDR, XDR (extensive drug resistance), and DTR (difficult-to-treat resistance) were calculated, in addition to the introduction of a composite multiple antibiotic resistance (pMAR) score and the modified versions (mDTR, mcDTR) of these resistance categories [33]. As there is limited data on the interplay between resistances to different antibiotic groups contributing to the multiple drug resistant phenotype, the aim of our study was to provide data in this regard from our already existing dataset, corresponding to UTIs in the Southern region of Hungary.

\section{Materials and Methods}

\subsection{Study Design, Setting}

In the present study, a secondary analysis of taxonomic and resistance data was performed, which was initially collected for epidemiological purposes [33]. The data corresponds to UTIs detected between the time period of 1 January 2008 to 31 December 2017 in outpatient clinics and inpatient departments at the Albert Szent-Györgyi Health Center, University of Szeged, a primary- and tertiary-care teaching hospital in the Southern Region of Hungary (serving 600,000 individuals in the region [34]). Data collection was performed manually from the database of the Department of Microbiology's (previously: Institute of Clinical Microbiology) laboratory information system (LIS), corresponding to urine samples positive for Gram-negative pathogens, based on the criteria described previously [33].

\subsection{Bacterial Identification, Antimicrobial Susceptibility Testing}

The processing of urine samples arriving at the Department of Medical Microbiology during the study period was carried out based on the relevant guidelines published by the Hungarian Ministry of Health [35]. Identification of isolates was carried out via classical biochemical tests, the VITEK 2 ID/AST (bioMérieux, Marcy-l'Étoile, France), and after 2012, matrix-assisted laser desorption/ionization time-of-flight mass spectrometry (MALDITOF MS) using a MicroFlex MALDI Biotyper (Bruker Daltonics, Bremen, Germany) [36]. Antimicrobial susceptibility testing for Gram-negative bacteria and the interpretation of the results was performed based on the recommendations of the European Committee on Antimicrobial Susceptibility Testing (EUCAST) at the time of isolation, taking into account the intrinsic resistance mechanisms of isolated bacteria [37]. Resistance to third-generation cephalosporins (3GCs) in Enterobacterales species was inferred from resistance against ceftriaxone and/or ceftazidime [38]. Intermediate results (I) for antibiotics other than 3GCs were grouped with and reported as resistant $(\mathrm{R})$ [36]. 


\subsection{Data Collection, Preparation for Data Analysis}

The data were collected from the LIS and saved as Microsoft Excel 2013 (Microsoft Corporation, Redmond, WA, USA) documents for statistical analysis. For the purposes of the analyses, the following taxonomic groups were set for Gram-negative bacteria: $E$. coli; Klebsiella spp.; the Citrobacter-Enterobacter-Serratia [CES] group; the Proteus-ProvidenciaMorganella [PPM] group; Acinetobacter spp.; and Pseudomonas spp. Additionally, indicator antibiotics were selected for each taxonomic group, for which, resistance data was included in the analysis. For Klebsiella spp., the CES group, and the PPM group, these antibiotics were 3GCs, ciprofloxacin (CIP), gentamicin (GEN), trimethoprim-sulfamethoxazole (SXT), and fosfomycin (FOS). For E. coli, they were 3GCs, CIP, GEN, SXT, FOS, and nitrofurantoin (NIT). For Acinetobacter spp., they were CIP, GEN, amikacin (AMI), SXT, imipenem (IMI), and meropenem (MER). For Pseudomonas spp., they were CIP, GEN, AMI, ceftazidime (CEFT), cefepime (CEFE), IMI, and MER. Due to the low number of carbapenem-resistant Enterobacterales, and the low number of colistin non-susceptible Gram-negative isolates overall, these antibiotics were not selected for the relevant taxonomic groups [36]. The following were identifiable from the data: year of isolation; outpatient/inpatient origin of the isolate; taxonomy; and susceptibility or resistance to indicator antibiotics (5-7, depending on the taxonomic group).

\subsection{Statistical Analyses: Principal Component Analysis (PCA), Pearson-Correlation}

Descriptive statistics was performed using Microsoft Excel 2013 (Microsoft Corporation, Redmond, WA, USA). The normality of variables was tested using the KolmogorovSmirnov test. Principal component analysis (PCA) was applied to assess the association between pair-wise variables (resistance against indicator antibiotics) in each taxonomic group. PCA was used to reduce data dimensions and to visualize each dataset in a two-dimensional space. During this process, the data was projected on new coordinate directions, i.e., principal component 1 [PC1] and principal component 2 [PC2], where the original data has the largest variance explained, to identify the presence of individual resistance in these bacteria and their contribution to overall resistance rates $[39,40]$. A correlation matrix was used to determine the association between the presence of resistance against indicator antibiotics. Based on the value of the Pearson-correlation coefficients (r), the relationship between the variables was determined as follows: $0.1<|\mathrm{r}|<0.3$ were denoted as weak correlation; $0.3<|\mathrm{r}|<0.5$ as moderate correlation; $0.5<|\mathrm{r}|<0.85$ as strong correlation; and $|\mathrm{r}| \geq 0.85$ as very strong correlation [41]. Statistical analyses were performed using the Past 4.01 statistical software (Paleontological Museum, University of Oslo; Oslo, Norway). $p$ values $<0.05$ were considered statistically significant.

\section{Results and Discussion}

\subsection{General Information}

Overall, data from $n=16,240$ outpatient and $n=13,964$ inpatient Gram-negative UTI isolates were included in the data analysis, corresponding to our 10-year (2008-2017) study period. The distribution among different taxonomic groups was the following $\left(\mathrm{n}_{\mathrm{OP}}\right.$ : outpatient; $\mathrm{n}_{\mathrm{IP}}$ : inpatient isolates): E. coli: $\mathrm{n}_{\mathrm{OP}}=12,002$ and $\mathrm{n}_{\mathrm{IP}}=8173$; Klebsiella spp.: $\mathrm{n}_{\mathrm{OP}}=1895$ and $\mathrm{n}_{\mathrm{IP}}=2$ 2952; CES group: $\mathrm{n}_{\mathrm{OP}}=554$ and $\mathrm{n}_{\mathrm{IP}}=578 ;$ PPM group: $\mathrm{n}_{\mathrm{OP}}=1058$ and $\mathrm{n}_{\mathrm{IP}}=1392$; Acinetobacter spp.: $\mathrm{n}_{\mathrm{OP}}=143$ and $\mathrm{n}_{\mathrm{IP}}=133$; and Pseudomonas spp.: $\mathrm{n}_{\mathrm{OP}}=588$ and $\mathrm{n}_{\mathrm{IP}}=1096$. The rates of MDR in the respective taxonomic groups were the following: E. coli 2.3\%; Klebsiella spp. 1.6\%; CES group 5.9\%; PPM group 9.1\%; Acinetobacter spp. 9.7\%; and Pseudomonas spp. 8.5\%. In most cases, inpatient isolates presented with significantly higher rates of resistance $(p<0.05)$ compared to outpatient isolates [33].

\subsection{Outpatient Isolates}

The results of the PCA analyses and the corresponding correlation matrices for the data from outpatient UTI isolates are presented in Figures 1-6 and Tables 1-6, respectively. In E. coli, strong positive correlations were seen between CIP-SXT $(\mathrm{r}=0.8474 ; p=0.002)$ 
and CIP-GEN resistance $(\mathrm{r}=0.6342 ; p=0.049)$, whereas strong negative correlation was shown for FOS and NIT resistance $(\mathrm{r}=-0.7183 ; p=0.019)$ (Figure 1, Table 1).

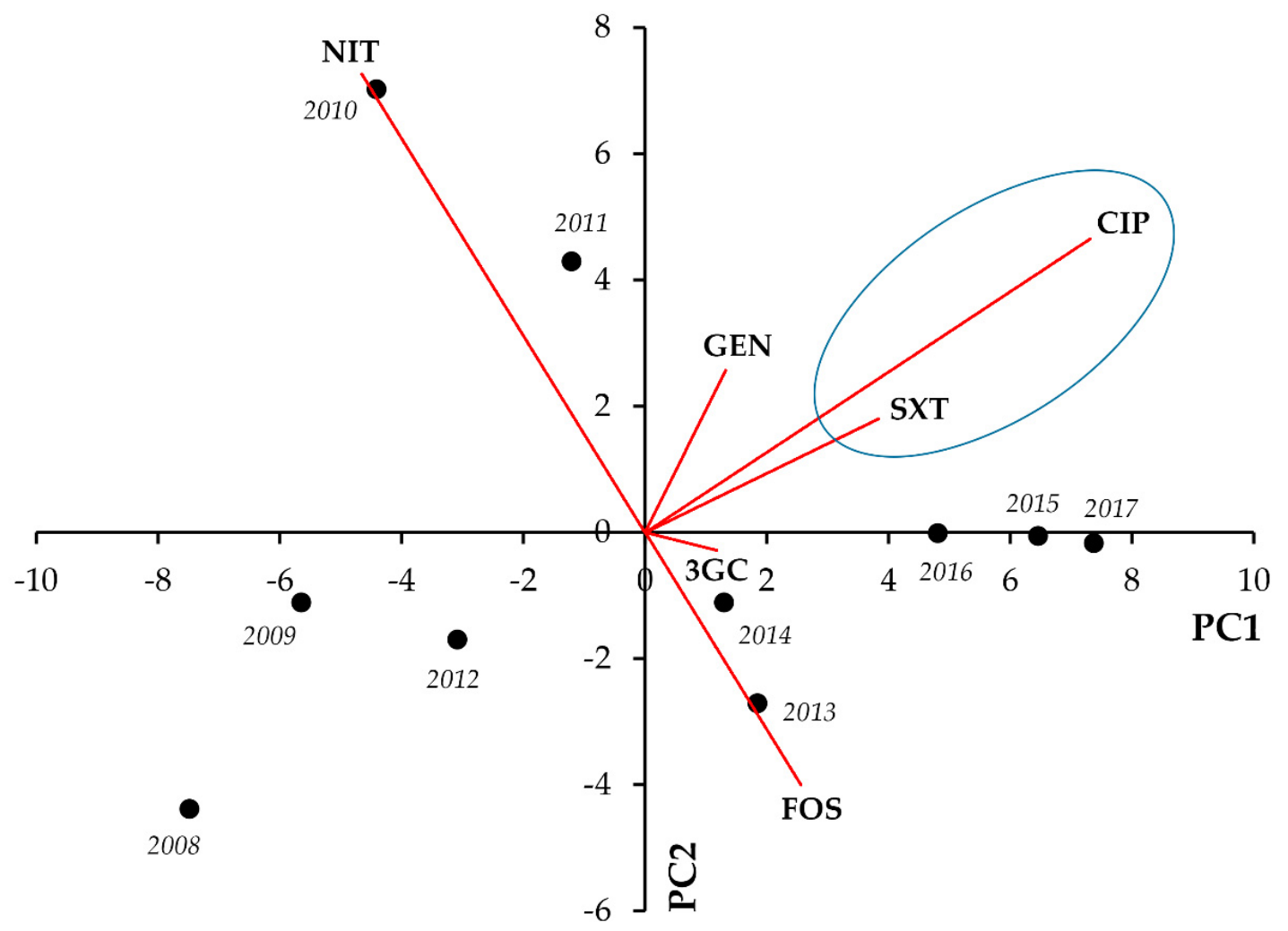

Figure 1. Principal component analysis (PCA) of resistance data for outpatient E. coli UTI isolates among six indicator antibiotics (2008-2017). PC1 and PC2 axes explained $62.05 \%$ and $25.50 \%$ of the total variance in the dataset, respectively. The blue ellipse denotes the positive correlation between the resistance to two antibiotics from a different antibiotic family with the highest Pearson-correlation coefficient.

Table 1. Correlation matrix of resistance data for outpatient E. coli UTI isolates among six indicator antibiotics (2008-2017).

\begin{tabular}{|c|c|c|c|c|c|c|c|}
\hline & CIP & NIT & GEN & SXT & $3 G C$ & FOS & \\
\hline CIP & $X$ & 0.2826 & 0.049 & 0.002 & 0.3693 & 0.3489 & \multirow{6}{*}{$\begin{array}{l}\text { Statistical } \\
\text { signifi- } \\
\text { cance } \\
(p=)\end{array}$} \\
\hline NIT & -0.3772 & $X$ & 0.7205 & 0.1918 & 0.1893 & 0.019 & \\
\hline GEN & 0.6342 & 0.1299 & $X$ & 0.051 & 0.6080 & 0.7434 & \\
\hline SXT & 0.8474 & -0.4501 & 0.6289 & $X$ & 0.078 & 0.4530 & \\
\hline $3 \mathrm{GC}$ & 0.3188 & -0.4524 & 0.1855 & 0.5798 & $X$ & 0.9514 & \\
\hline FOS & 0.3318 & -0.7183 & 0.1189 & 0.2686 & -0.022 & $X$ & \\
\hline
\end{tabular}

Green cells denote Pearson-correlation coefficients representing strong $(0.5<|\mathrm{r}|<0.85)$ and statistically significant $(p<0.05)$ correlations; blue cells denote Pearson-correlation coefficients representing strong $(0.5<|\mathrm{r}|<0.85)$, but statistically non-significant $(p \geq 0.05)$ correlations; white cells denote Pearson-correlation coefficients representing moderate or weak $(|\mathrm{r}|<0.5)$, statistically non-significant $(p \geq 0.05)$ correlations. Legend: CIP: ciprofloxacin; NIT: nitrofurantoin; GEN: gentamicin; SXT: trimethoprim-sulfamethoxazole; 3GC: third-generation cephalosporin; FOS: fosfomycin.

In Klebsiella spp., strong positive correlation was seen between CIP-3GC ( $\mathrm{r}=0.7976$; $p=0.006)$ and CIP-GEN resistance $(\mathrm{r}=0.7428 ; p=0.014)$ (Figure 2, Table 2). 


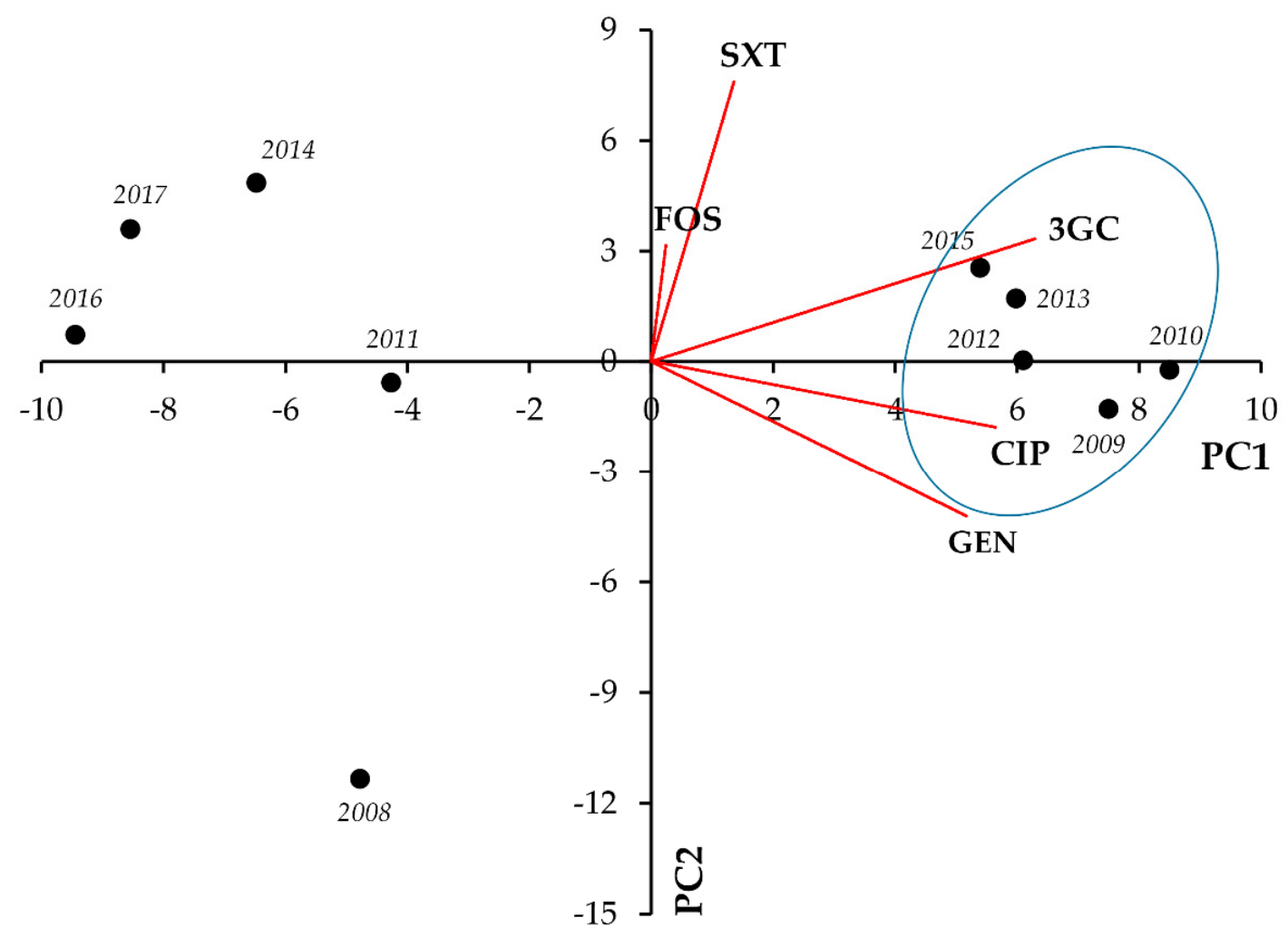

Figure 2. Principal component analysis (PCA) of resistance data for outpatient Klebsiella spp. UTI isolates among five indicator antibiotics (2008-2017). PC1 and PC2 axes explained 57.23\% and $21.28 \%$ of the total variance in the dataset, respectively. The blue ellipse denotes the positive correlation between the resistance to two antibiotics from a different antibiotic family with the highest Pearson-correlation coefficient.

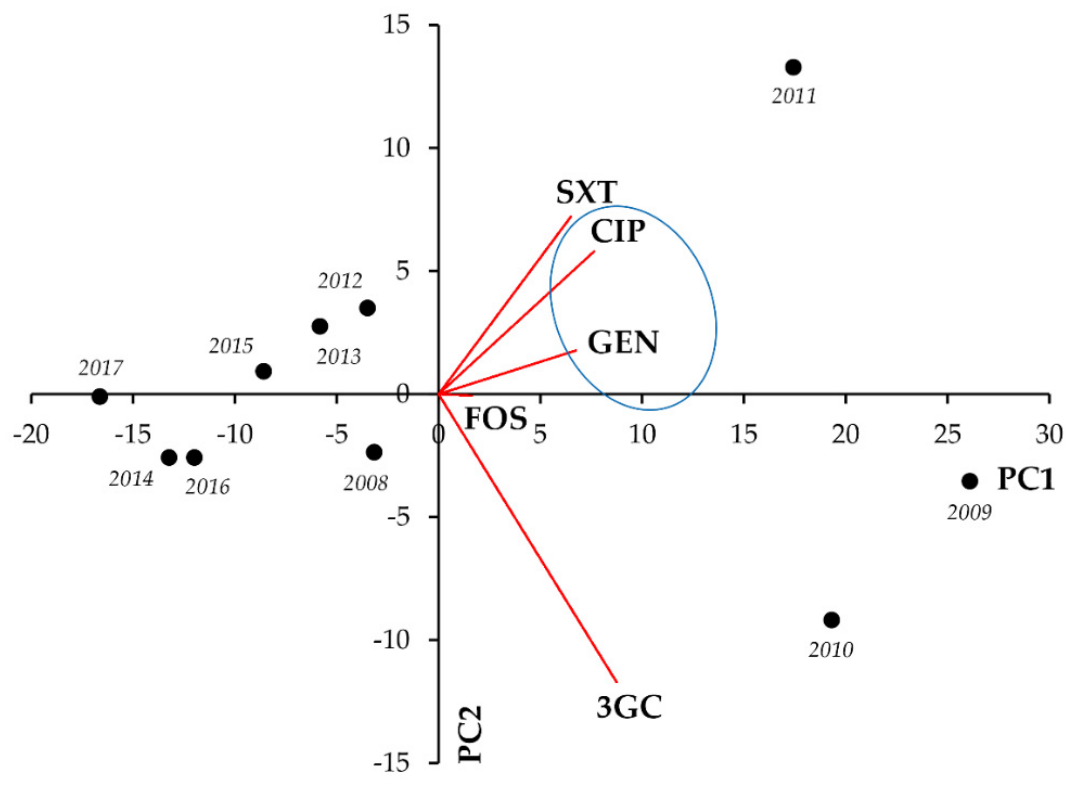

Figure 3. Principal component analysis (PCA) of resistance data for outpatient CES group UTI isolates among five indicator antibiotics (2008-2017). PC1 and PC2 axes explained $79.39 \%$ and $11.91 \%$ of the total variance in the dataset, respectively. The blue ellipse denotes the positive correlation between the resistance to two antibiotics from a different antibiotic family with the highest Pearson-correlation coefficient. 


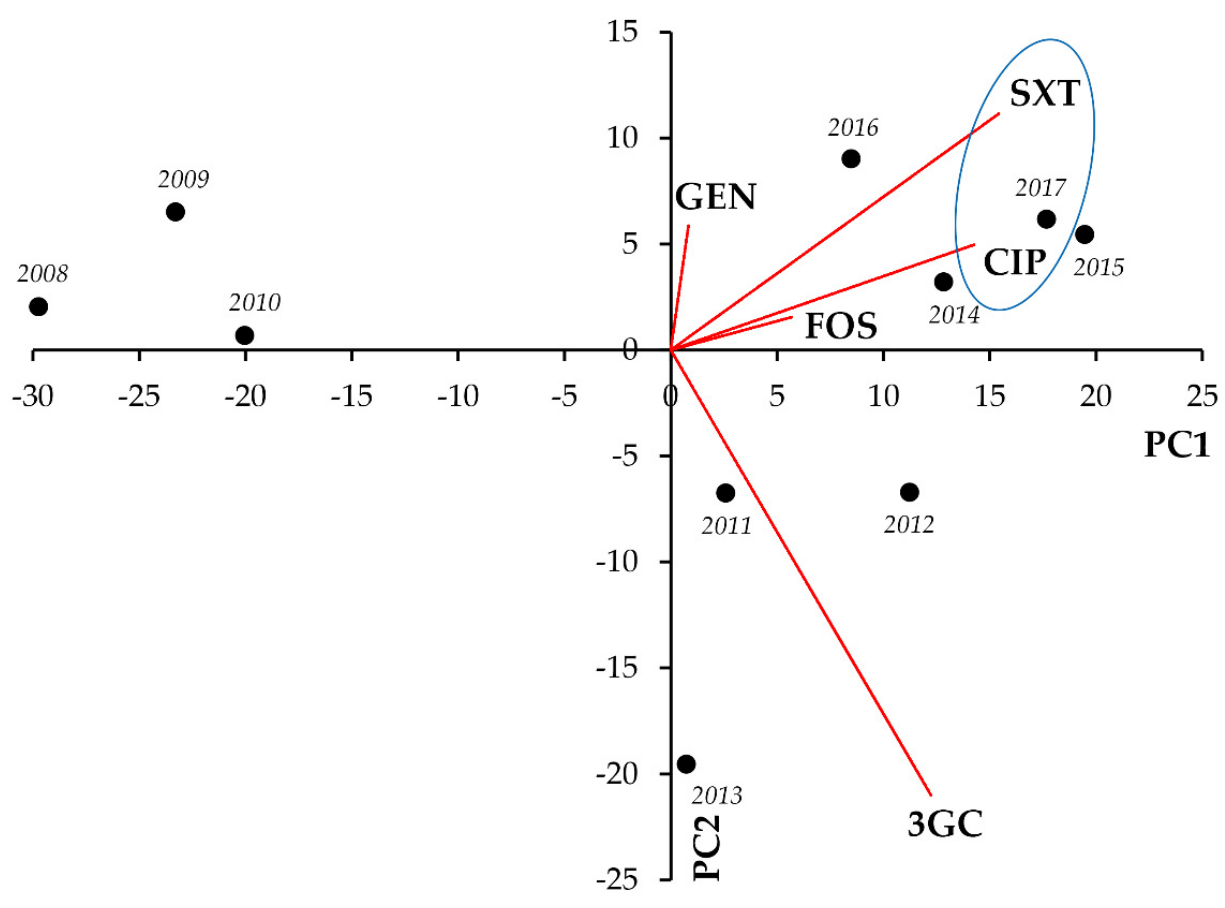

Figure 4. Principal component analysis (PCA) of resistance data for outpatient PPM group UTI isolates among five indicator antibiotics (2008-2017). PC1 and PC2 axes explained $77.01 \%$ and $18.09 \%$ of the total variance in the dataset, respectively. The blue ellipse denotes the positive correlation between the resistance to two antibiotics from a different antibiotic family with the highest Pearsoncorrelation coefficient.

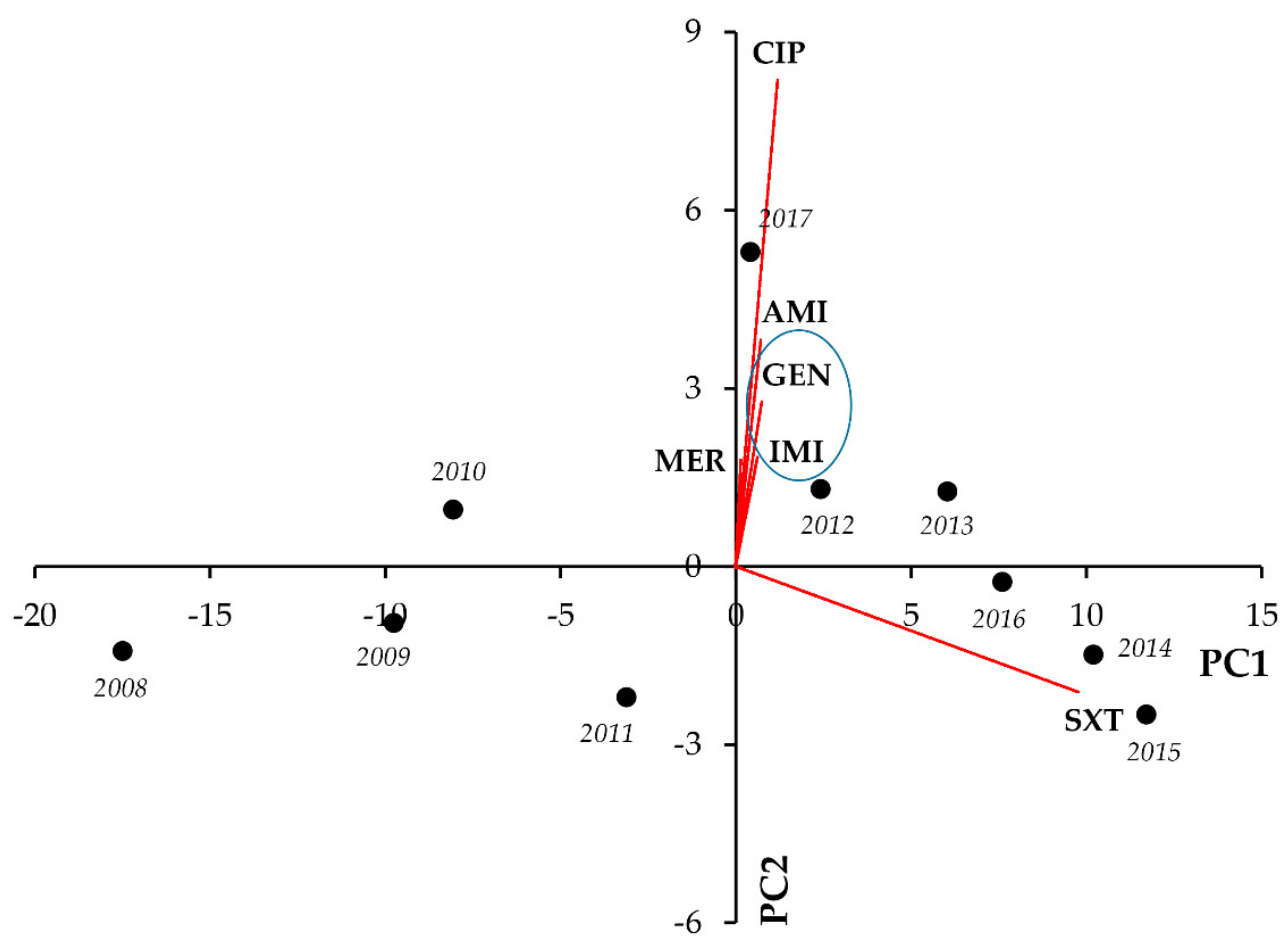

Figure 5. Principal component analysis (PCA) of resistance data for outpatient Acinetobacter spp. UTI isolates among six indicator antibiotics (2008-2017). PC1 and PC2 axes explained $92.66 \%$ and $5.50 \%$ of the total variance in the dataset, respectively. The blue ellipse denotes the positive correlation between the resistance to two antibiotics from a different antibiotic family with the highest Pearson-correlation coefficient. 


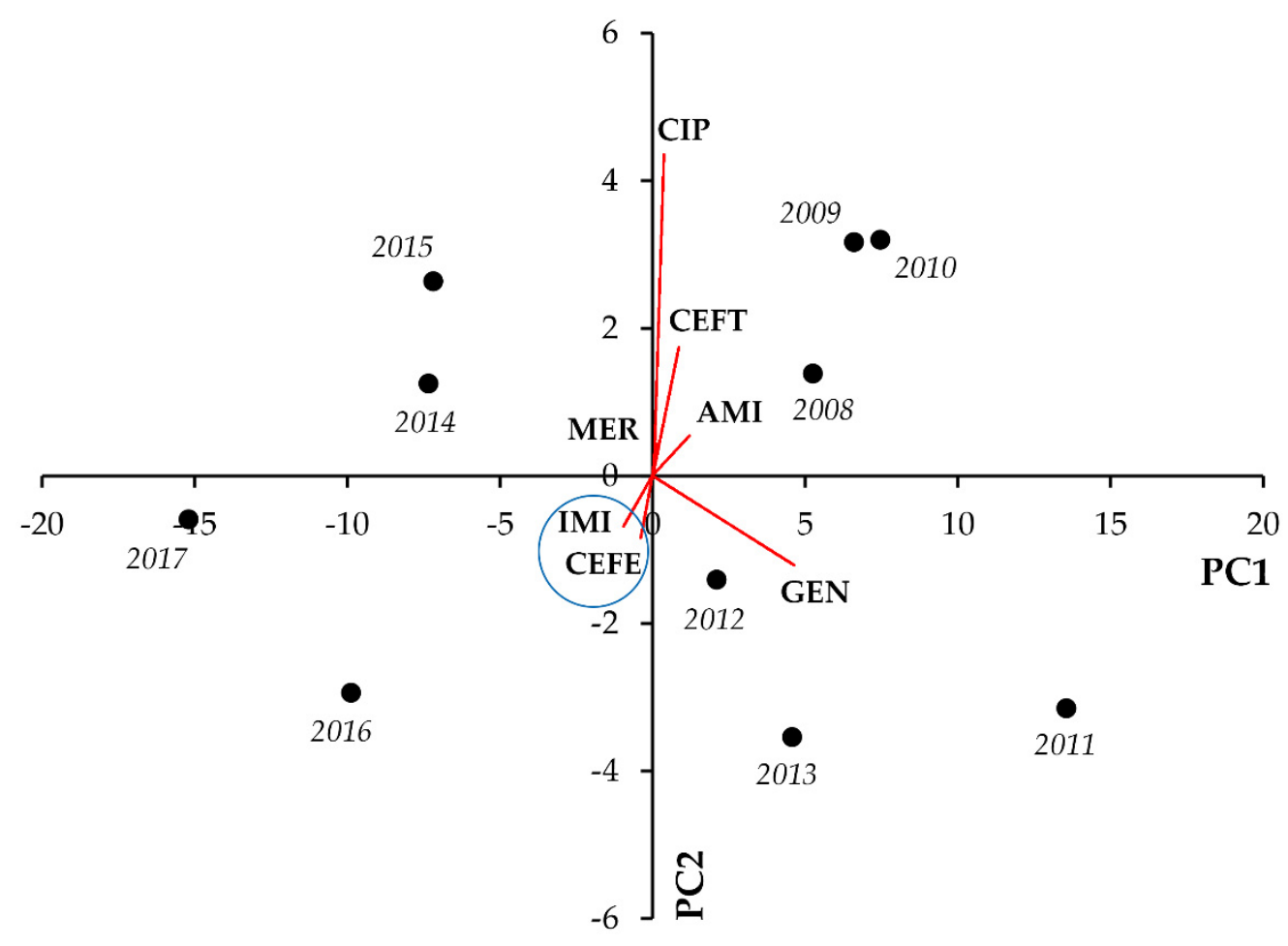

Figure 6. Principal component analysis (PCA) of resistance data for outpatient Pseudomonas spp. UTI isolates among seven indicator antibiotics (2008-2017). PC1 and PC2 axes explained $85.56 \%$ and $7.12 \%$ of the total variance in the dataset, respectively. The blue ellipse denotes the positive correlation between the resistance to two antibiotics from a different antibiotic family with the highest Pearson-correlation coefficient.

Table 2. Correlation matrix of resistance data for outpatient Klebsiella spp. UTI isolates among five indicator antibiotics (2008-2017).

\begin{tabular}{|c|c|c|c|c|c|c|}
\hline & CIP & GEN & SXT & 3GC & FOS & \\
\hline CIP & $X$ & 0.014 & 0.9653 & 0.006 & 0.8964 & \multirow{5}{*}{$\begin{array}{l}\text { Statistical } \\
\text { signifi- } \\
\text { cance } \\
(p=)\end{array}$} \\
\hline GEN & 0.7428 & $X$ & 0.8188 & 0.1723 & 0.4207 & \\
\hline SXT & 0.0158 & 0.0834 & $X$ & 0.4319 & 0.7445 & \\
\hline 3GC & 0.7976 & 0.4679 & 0.2808 & $X$ & 0.4336 & \\
\hline FOS & 0.0475 & -0.2874 & 0.1184 & 0.2798 & $X$ & \\
\hline
\end{tabular}

Green cells denote Pearson-correlation coefficients representing strong $(0.5<|\mathrm{r}|<0.85)$ and statistically significant $(p<0.05)$ correlations; white cells denote Pearson-correlation coefficients representing moderate or weak $(|r|<0.5)$, statistically non-significant $(p \geq 0.05)$ correlations. Legend: CIP: ciprofloxacin; GEN: gentamicin; SXT: trimethoprim-sulfamethoxazole; 3GC: third-generation cephalosporin; FOS: fosfomycin.

For members of the Citrobacter-Enterobacter-Serratia group, the existence of resistance to most indicator antibiotics showed strong and significant positive correlations (the strongest being GEN-CIP [ $\mathrm{r}=0.9326 ; p<0.001]$, GEN-SXT [ $\mathrm{r}=0.8152 ; p=0.004]$, and GEN-3GC $[\mathrm{r}=0.8144 ; p=0.004]$, respectively), whereas such strong associations were not shown between resistance to FOS and other antimicrobials (Figure 3, Table 3).

For members of the Proteus-Providencia-Morganella group, strong and significant positive correlations were seen between FOS-CIP $(r=0.8656 ; p<0.001)$, FOS-SXT $(r=0.8656$; $p<0.001)$, and CIP-SXT $(\mathrm{r}=0.9251 ; p<0.001)$ (Figure 4, Table 4). 
Table 3. Correlation matrix of resistance data for outpatient CES group UTI isolates among five indicator antibiotics (2008-2017).

\begin{tabular}{|c|c|c|c|c|c|c|}
\hline & CIP & 3GC & GEN & SXT & FOS & \\
\hline CIP & $X$ & 0.035 & $<0.001$ & 0.017 & 0.3837 & \multirow{5}{*}{$\begin{array}{l}\text { Statistical } \\
\text { signifi- } \\
\text { cance } \\
(p=)\end{array}$} \\
\hline 3GC & 0.6683 & $X$ & 0.004 & 0.068 & 0.1181 & \\
\hline GEN & 0.9326 & 0.8144 & $X$ & 0.004 & 0.1524 & \\
\hline SXT & 0.7266 & 0.5974 & 0.8152 & $X$ & 0.027 & \\
\hline FOS & 0.3098 & 0.5263 & 0.4881 & 0.6895 & $X$ & \\
\hline
\end{tabular}

Orange cells denote Pearson-correlation coefficients representing very strong ( $|\mathrm{r}| \geq 0.85)$ and statistically significant $(p<0.05)$ correlations; green cells denote Pearson-correlation coefficients representing strong $(0.5<|\mathrm{r}|<0.85)$ and statistically significant $(p<0.05)$ correlations; blue cells denote Pearson-correlation coefficients representing strong $(0.5<|\mathrm{r}|<0.85)$, but statistically non-significant $(p \geq 0.05)$ correlations; white cells denote Pearson-correlation coefficients representing moderate or weak $(|\mathrm{r}|<0.5)$, statistically non-significant $(p \geq 0.05)$ correlations. Legend: CIP: ciprofloxacin; GEN: gentamicin; SXT: trimethoprim-sulfamethoxazole; 3GC: third-generation cephalosporin; FOS: fosfomycin.

Table 4. Correlation matrix of resistance data for outpatient PPM group UTI isolates among five indicator antibiotics (2008-2017).

\begin{tabular}{|c|c|c|c|c|c|c|}
\hline & CIP & 3GC & GEN & SXT & FOS & \\
\hline CIP & $X$ & 0.051 & 0.4931 & $<0.001$ & $<0.001$ & \multirow{5}{*}{$\begin{array}{l}\text { Statistical } \\
\text { signifi- } \\
\text { cance } \\
(p=)\end{array}$} \\
\hline 3GC & 0.6296 & $X$ & 0.5959 & 0.1318 & 0.051 & \\
\hline GEN & 0.2461 & -0.1916 & $X$ & 0.4162 & 0.4188 & \\
\hline SXT & 0.9251 & 0.5103 & 0.2901 & $X$ & $<0.001$ & \\
\hline FOS & 0.8656 & 0.6304 & 0.2886 & 0.8992 & $X$ & \\
\hline
\end{tabular}

Orange cells denote Pearson-correlation coefficients representing very strong ( $|r| \geq 0.85)$ and statistically significant $(p<0.05)$ correlations; blue cells denote Pearson-correlation coefficients representing strong $(0.5<|\mathrm{r}|$ $<0.85)$, but statistically non-significant $(p \geq 0.05)$ correlations; white cells denote Pearson-correlation coefficients representing moderate or weak $(|\mathrm{r}|<0.5)$, statistically non-significant $(p \geq 0.05)$ correlations. Legend: CIP: ciprofloxacin; GEN: gentamicin; SXT: trimethoprim-sulfamethoxazole; 3GC: third-generation cephalosporin; FOS: fosfomycin.

Table 5. Correlation matrix of resistance data for outpatient Acinetobacter spp. UTI isolates among six indicator antibiotics (2008-2017).

\begin{tabular}{|c|c|c|c|c|c|c|c|}
\hline & CIP & IMI & MER & GEN & AMI & SXT & \\
\hline CIP & $X$ & 0.041 & 0.039 & 0.026 & 0.019 & 0.1009 & \multirow{6}{*}{$\begin{array}{l}\text { Statistical } \\
\text { signifi- } \\
\text { cance } \\
(p=)\end{array}$} \\
\hline IMI & 0.6507 & $X$ & 0.2298 & 0.001 & 0.002 & 0.054 & \\
\hline MER & 0.6554 & 0.4176 & $X$ & 0.4330 & 0.3727 & 0.7539 & \\
\hline GEN & 0.6945 & 0.8620 & 0.2802 & $X$ & $<0.001$ & 0.048 & \\
\hline AMI & 0.7159 & 0.8375 & 0.3166 & 0.9838 & $X$ & 0.035 & \\
\hline SXT & 0.5482 & 0.6233 & 0.1139 & 0.6361 & 0.6669 & $X$ & \\
\hline
\end{tabular}

Orange cells denote Pearson-correlation coefficients representing very strong $(|r| \geq 0.85)$ and statistically significant $(p<0.05)$ correlations; green cells denote Pearson-correlation coefficients representing strong $(0.5<|\mathrm{r}|<0.85)$ and statistically significant $(p<0.05)$ correlations; blue cells denote Pearson-correlation coefficients representing strong $(0.5<|\mathrm{r}|<0.85)$, but statistically non-significant $(p \geq 0.05)$ correlations; white cells denote Pearsoncorrelation coefficients representing moderate or weak $(|\mathrm{r}|<0.5)$, statistically non-significant $(p \geq 0.05)$ correlations. Legend: CIP: ciprofloxacin; IMI: imipenem; MER: meropenem; GEN: gentamicin; AMI: amikacin; SXT: trimethoprim-sulfamethoxazole.

In the case of Acinetobacter spp., CIP showed strong and significant positive correlations with most indicator antibiotics (apart from SXT, which showed strong positive associations with the aminoglycosides GEN and AMI). In addition, GEN-IMI and GENAMI co-resistance presented with very strong and significant correlations $(r=0.8620$, $p=0.001$, and $\mathrm{r}=0.9838, p<0.001$, respectively) (Figure 5, Table 5). 
Table 6. Correlation matrix of resistance data for outpatient Pseudomonas spp. UTI isolates among seven indicator antibiotics (2008-2017).

\begin{tabular}{|c|c|c|c|c|c|c|c|c|}
\hline & CIP & IMI & MER & CEFT & CEFE & GEN & AMI & \\
\hline CIP & $X$ & 0.5507 & 0.3572 & 0.1489 & 0.1359 & 0.3528 & 0.1145 & \multirow{7}{*}{$\begin{array}{l}\text { Statistical } \\
\text { signifi- } \\
\text { cance } \\
(p=)\end{array}$} \\
\hline IMI & -0.2151 & $X$ & 0.0991 & 0.007 & 0.001 & 0.038 & 0.3386 & \\
\hline MER & 0.3265 & -0.5505 & $X$ & 0.1316 & 0.016 & 0.3288 & 0.5279 & \\
\hline CEFT & -0.4917 & -0.7879 & -0.5105 & $X$ & 0.002 & 0.063 & 0.1911 & \\
\hline CEFE & 0.5057 & 0.8617 & 0.7338 & -0.8376 & $X$ & 0.028 & 0.1139 & \\
\hline GEN & 0.3293 & -0.6595 & 0.3451 & -0.6056 & 0.6857 & $X$ & $<0.001$ & \\
\hline AMI & 0.5307 & -0.3386 & 0.2272 & -0.4507 & 0.5315 & 0.8745 & $X$ & \\
\hline
\end{tabular}

Orange cells denote Pearson-correlation coefficients representing very strong $(|\mathrm{r}| \geq 0.85)$ and statistically significant $(p<0.05)$ correlations; green cells denote Pearson-correlation coefficients representing strong $(0.5<|\mathrm{r}|<0.85)$ and statistically significant $(p<0.05)$ correlations; blue cells denote Pearson-correlation coefficients representing strong $(0.5<|\mathrm{r}|<0.85)$, but statistically non-significant $(p \geq 0.05)$ correlations; white cells denote Pearsoncorrelation coefficients representing moderate or weak $(|\mathrm{r}|<0.5)$, statistically non-significant $(p \geq 0.05)$ correlations. Legend: CIP: ciprofloxacin; IMI: imipenem; MER: meropenem; CEFT: ceftazidime; CEFE: cefepime; GEN: gentamicin; AMI: amikacin.

For Pseudomonas spp., most notable positive correlations were seen between CEFE and IMI $(\mathrm{r}=0.8617 ; p=0.001), \operatorname{MER}(\mathrm{r}=0.7338 ; p=0.016)$ and GEN $(\mathrm{r}=0.6857$; $p=0.028)$ resistance, and GEN-AMI resistance $(\mathrm{r}=0.8745 ; p<0.001)$. On the other hand, strong negative correlations arose among CEFT-IMI $(\mathrm{r}=-0.7879 ; p=0.007)$, CEFT-CEFE $(\mathrm{r}=-0.8376 ; p=0.002)$, and GEN-IMI $(\mathrm{r}=-0.6595 ; p=0.038)$ (Figure 6, Table 6).

\subsection{Inpatient Isolates}

The results of the PCA analyses and the corresponding correlation matrices for the data from inpatient UTI isolates are presented in Figures 7-12 and Tables 7-12, respectively. Similarly to the case of outpatient isolates, strong positive correlation was seen between CIP-GEN ( $\mathrm{r}=0.9602 ; p<0.001)$, CIP-3GC $(\mathrm{r}=0.7476 ; p=0.012)$, and GEN-3GC $(\mathrm{r}=0.8099 ; p=0.004)$, whereas strong negative correlation was seen for FOS and NIT resistance $(\mathrm{r}=-0.7437 ; p=0.014)$ (Figure 7, Table 7).

Table 7. Correlation matrix of resistance data for inpatient E. coli UTI isolates among six indicator antibiotics (2008-2017).

\begin{tabular}{|c|c|c|c|c|c|c|c|}
\hline & CIP & NIT & GEN & SXT & 3GC & FOS & \\
\hline CIP & $X$ & 0.1057 & $<0.001$ & 0.1865 & 0.012 & 0.2239 & \multirow{6}{*}{$\begin{array}{l}\text { Statistical } \\
\text { signifi- } \\
\text { cance } \\
(p=)\end{array}$} \\
\hline NIT & -0.5418 & $X$ & 0.1449 & 0.0942 & 0.1694 & 0.014 & \\
\hline GEN & 0.9602 & -0.4959 & $X$ & 0.1503 & 0.004 & 0.3587 & \\
\hline SXT & 0.4549 & -0.5572 & 0.4902 & $X$ & 0.068 & 0.2854 & \\
\hline $3 \mathrm{GC}$ & 0.7476 & -0.4711 & 0.8099 & 0.5970 & $X$ & 0.6951 & \\
\hline FOS & 0.4224 & -0.7437 & 0.3255 & 0.3752 & 0.1422 & $X$ & \\
\hline
\end{tabular}

Orange cells denote Pearson-correlation coefficients representing very strong $(|\mathrm{r}| \geq 0.85)$ and statistically significant $(p<0.05)$ correlations; green cells denote Pearson-correlation coefficients representing strong $(0.5<|\mathrm{r}|<0.85)$ and statistically significant $(p<0.05)$ correlations; blue cells denote Pearson-correlation coefficients representing strong $(0.5<|\mathrm{r}|<0.85)$, but statistically non-significant $(p \geq 0.05)$ correlations; white cells denote Pearsoncorrelation coefficients representing moderate or weak $(|\mathrm{r}|<0.5)$, statistically non-significant $(p \geq 0.05)$ correlations. Legend: CIP: ciprofloxacin; NIT: nitrofurantoin; GEN: gentamicin; SXT: trimethoprim-sulfamethoxazole 3GC: third-generation cephalosporin; FOS: fosfomycin.

In Klebsiella spp., strong positive correlation was seen between resistance to CIP-SXT $(\mathrm{r}=0.8144 ; p=0.004)$ and CIP-FOS $(\mathrm{r}=0.7758 ; p<0.001)$ (Figure 8, Table 8). 


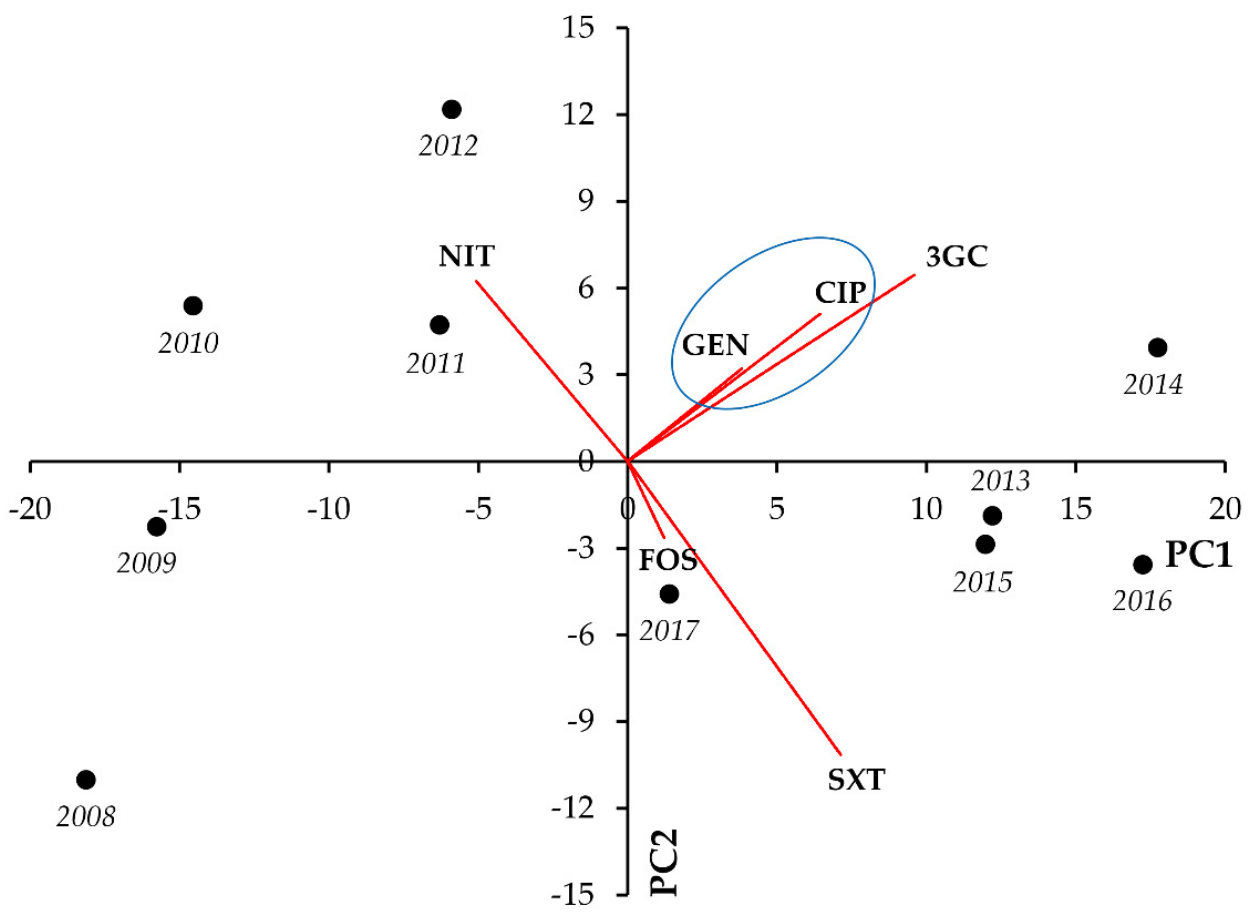

Figure 7. Principal component analysis (PCA) of resistance data for inpatient E. coli UTI isolates among six indicator antibiotics (2008-2017). PC1 and PC2 axes explained $68.39 \%$ and $14.97 \%$ of the total variance in the dataset, respectively. The blue ellipse denotes the positive correlation between the resistance to two antibiotics from a different antibiotic family with the highest Pearson-correlation coefficient.

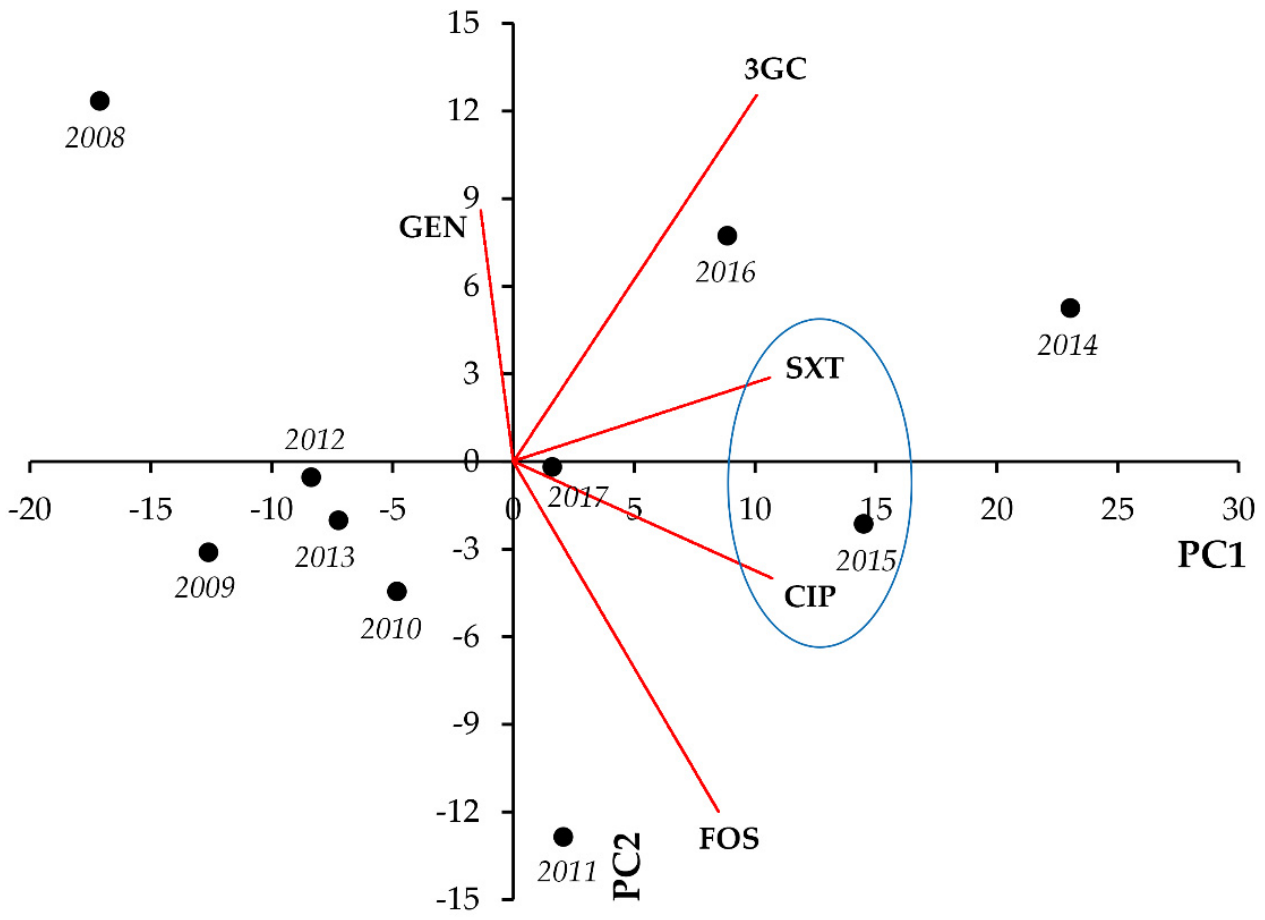

Figure 8. Principal component analysis (PCA) of resistance data for inpatient Klebsiella spp. UTI isolates among five indicator antibiotics (2008-2017). PC1 and PC2 axes explained $66.42 \%$ and $20.71 \%$ of the total variance in the dataset, respectively. The blue ellipse denotes the positive correlation between the resistance to two antibiotics from a different antibiotic family with the highest Pearson-correlation coefficient. 


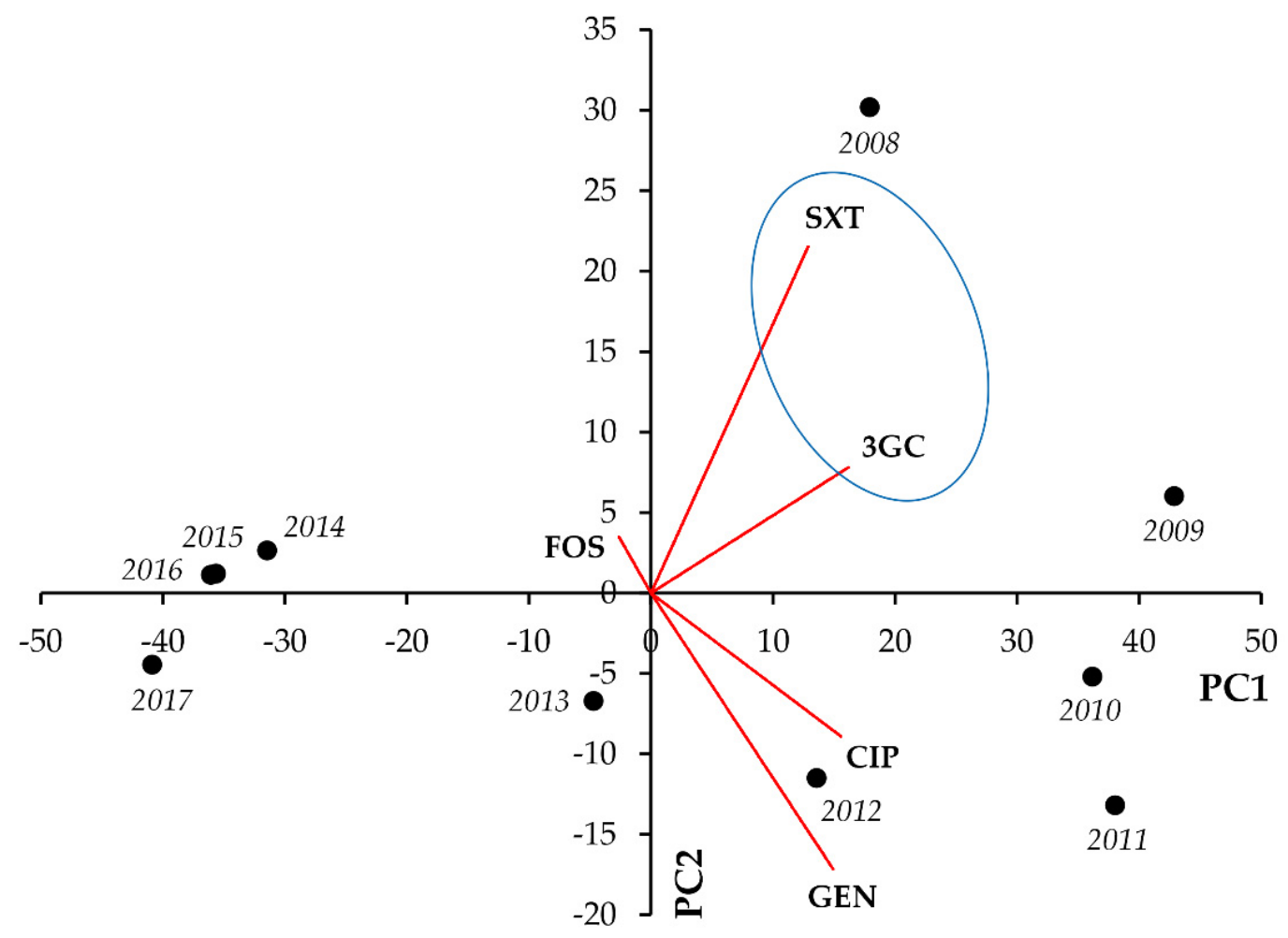

Figure 9. Principal component analysis (PCA) of resistance data for inpatient CES group UTI isolates among five indicator antibiotics (2008-2017). PC1 and PC2 axes explained $81.49 \%$ and $10.67 \%$ of the total variance in the dataset, respectively. The blue ellipse denotes the positive correlation between the resistance to two antibiotics from a different antibiotic family with the highest Pearson-correlation coefficient.

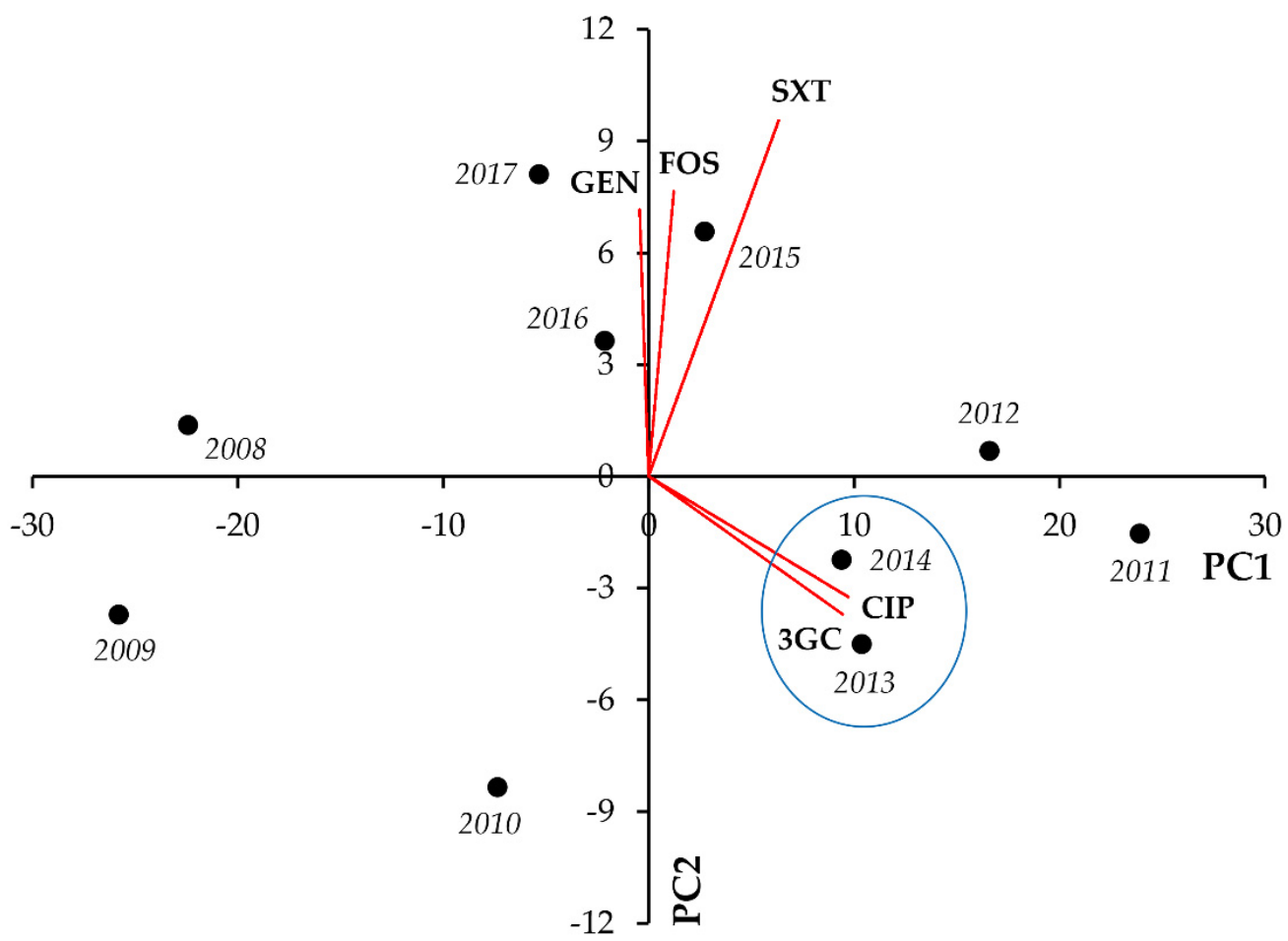

Figure 10. Principal component analysis (PCA) of resistance data for inpatient PPM group UTI isolates among five indicator antibiotics (2008-2017). PC1 and PC2 axes explained $81.85 \%$ and $8.35 \%$ of the total variance in the dataset, respectively. The blue ellipse denotes the positive correlation between the resistance to two antibiotics from a different antibiotic family with the highest Pearson-correlation coefficient. 


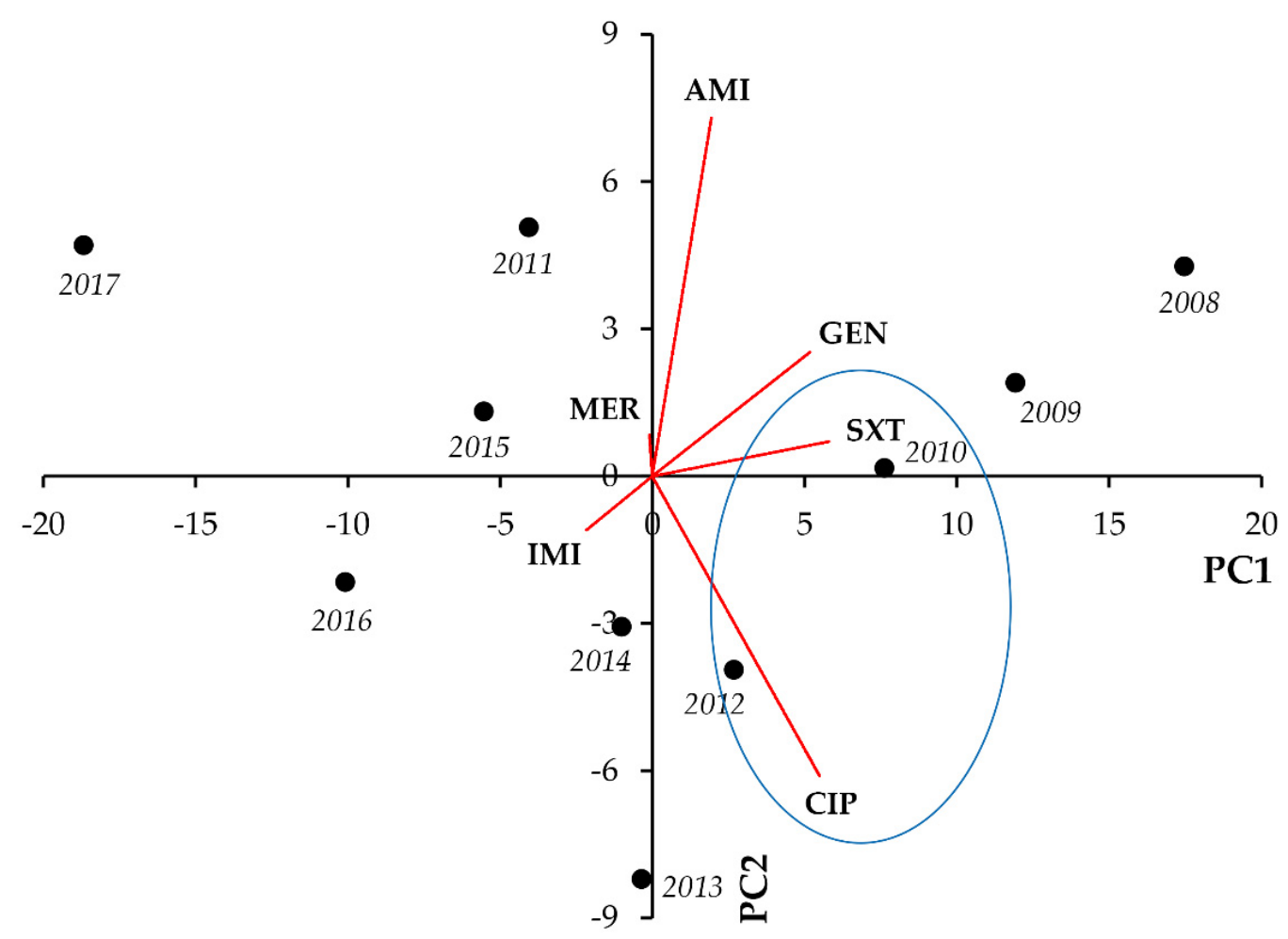

Figure 11. Principal component analysis (PCA) of resistance data for inpatient Acinetobacter spp. UTI isolates among six indicator antibiotics (2008-2017). PC1 and PC2 axes explained $75.02 \%$ and $12.51 \%$ of the total variance in the dataset, respectively. The blue ellipse denotes the positive correlation between the resistance to two antibiotics from a different antibiotic family with the highest Pearson-correlation coefficient.

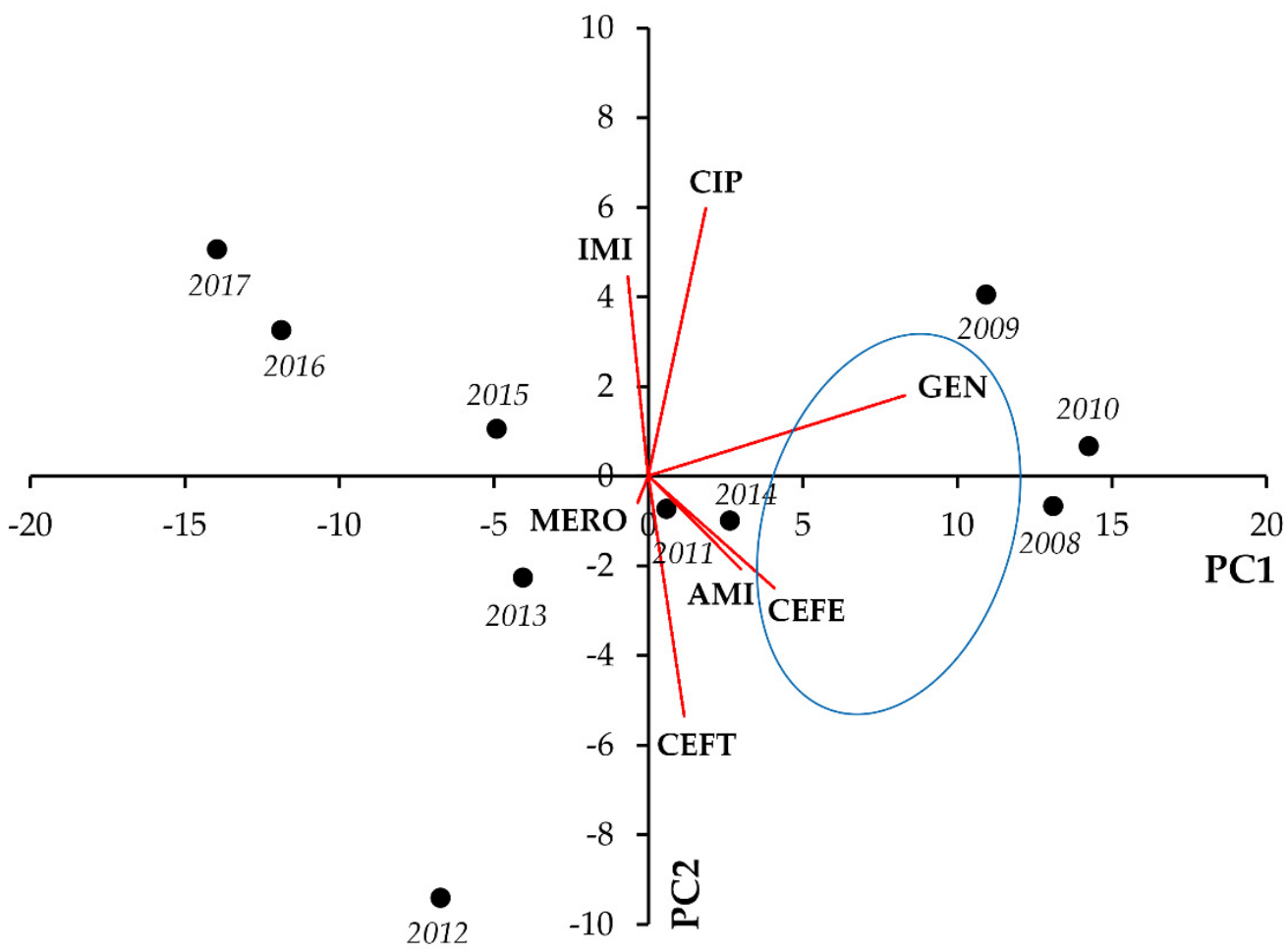

Figure 12. Principal component analysis (PCA) of resistance data for inpatient Pseudomonas spp. UTI isolates among seven indicator antibiotics (2008-2017). PC1 and PC2 axes explained $74.41 \%$ and $12.08 \%$ of the total variance in the dataset, respectively. The blue ellipse denotes the positive correlation between the resistance to two antibiotics from a different antibiotic family with the highest Pearson-correlation coefficient. 
Table 8. Correlation matrix of resistance data for inpatient Klebsiella spp. UTI isolates among five indicator antibiotics (2008-2017).

\begin{tabular}{|c|c|c|c|c|c|c|}
\hline & CIP & GEN & SXT & 3GC & FOS & \\
\hline CIP & $\mathrm{X}$ & 0.2592 & 0.004 & 0.060 & $<0.001$ & \multirow{5}{*}{$\begin{array}{l}\text { Statistical } \\
\text { signifi- } \\
\text { cance } \\
(p=)\end{array}$} \\
\hline GEN & -0.3946 & $X$ & 0.9287 & 0.5970 & 0.092 & \\
\hline SXT & 0.8144 & -0.0326 & $x$ & 0.054 & 0.1501 & \\
\hline 3GC & 0.6119 & 0.1910 & 0.6240 & $X$ & 0.3655 & \\
\hline FOS & 0.7758 & -0.5599 & 0.4904 & 0.3212 & $X$ & \\
\hline
\end{tabular}

Green cells denote Pearson-correlation coefficients representing strong $(0.5<|\mathrm{r}|<0.85)$ and statistically significant $(p<0.05)$ correlations; white cells denote Pearson-correlation coefficients representing moderate or weak $(|\mathrm{r}|<0.5)$, statistically non-significant $(p \geq 0.05)$ correlations. Legend: CIP: ciprofloxacin; GEN: gentamicin; SXT: trimethoprim-sulfamethoxazole; 3GC: third-generation cephalosporin; FOS: fosfomycin.

Table 9. Correlation matrix of resistance data for inpatient CES group UTI isolates among five indicator antibiotics (2008-2017).

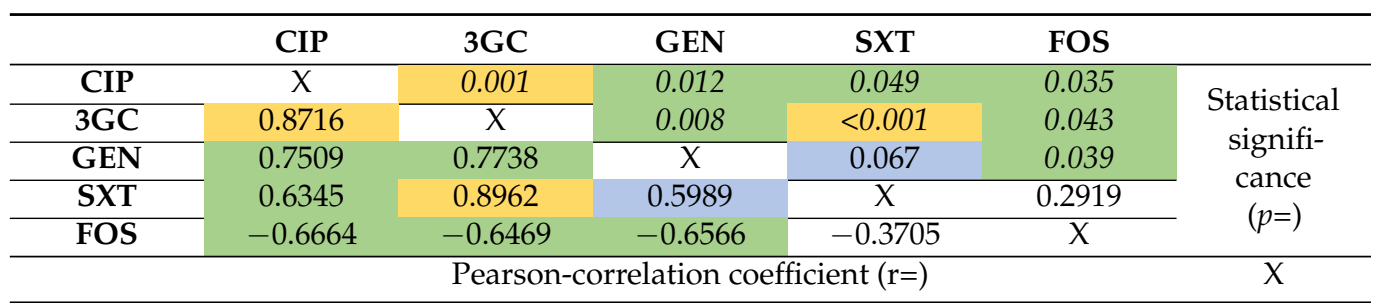

Orange cells denote Pearson-correlation coefficients representing very strong $(|\mathrm{r}| \geq 0.85)$ and statistically significant $(p<0.05)$ correlations; green cells denote Pearson-correlation coefficients representing strong $(0.5<|\mathrm{r}|<0.85)$ and statistically significant $(p<0.05)$ correlations; blue cells denote Pearson-correlation coefficients representing strong $(0.5<|\mathrm{r}|<0.85)$, but statistically non-significant $(p \geq 0.05)$ correlations; white cells denote Pearsoncorrelation coefficients representing moderate or weak $(|\mathrm{r}|<0.5)$, statistically non-significant $(p \geq 0.05)$ correlations. Legend: CIP: ciprofloxacin; GEN: gentamicin; SXT: trimethoprim-sulfamethoxazole; 3GC: third-generation cephalosporin; FOS: fosfomycin.

Table 10. Correlation matrix of resistance data for inpatient PPM group UTI isolates among five indicator antibiotics (2008-2017).

\begin{tabular}{|c|c|c|c|c|c|c|}
\hline & CIP & 3GC & GEN & SXT & FOS & \multirow{6}{*}{$\begin{array}{l}\text { Statistical } \\
\text { signifi- } \\
\text { cance } \\
\quad(p=)\end{array}$} \\
\hline CIP & $X$ & $<0.001$ & 0.6076 & 0.005 & 0.4827 & \\
\hline 3GC & 0.8921 & $\mathrm{X}$ & 0.8589 & 0.015 & 0.3256 & \\
\hline GEN & -0.1857 & -0.0648 & $\mathrm{X}$ & 0.9475 & 0.2545 & \\
\hline SXT & 0.8007 & 0.7373 & -0.024 & $X$ & 0.068 & \\
\hline FOS & 0.2519 & 0.3472 & 0.3982 & 0.5966 & $X$ & \\
\hline \multicolumn{6}{|c|}{ Pearson-correlation coefficient $(\mathrm{r}=)$} & $\mathrm{X}$ \\
\hline
\end{tabular}

Orange cells denote Pearson-correlation coefficients representing very strong $(|\mathrm{r}| \geq 0.85)$ and statistically significant $(p<0.05)$ correlations; blue cells denote Pearson-correlation coefficients representing strong $(0.5<|\mathrm{r}|<0.85)$, but statistically non-significant $(p \geq 0.05)$ correlations; white cells denote Pearson-correlation coefficients representing moderate or weak $(|\mathrm{r}|<0.5)$, statistically non-significant $(p \geq 0.05)$ correlations. Legend: CIP: ciprofloxacin; GEN: gentamicin; SXT: trimethoprim-sulfamethoxazole; 3GC: third-generation cephalosporin; FOS: fosfomycin.

For members of the Citrobacter-Enterobacter-Serratia group, the resistance to one indicator antibiotic showed a strong correlation with resistance to all other indicator antibiotics in almost all cases (strongest correlation was observed for 3GC-CIP [ $\mathrm{r}=0.8716 ; p=0.001]$ and 3GC-SXT [ $r=0.8962 ; p<0.001]$, respectively). Nonetheless, correlations among FOS and the other antimicrobials were exclusively negative (Figure 9, Table 9).

In case of the Proteus-Providencia-Morganella group, strong and significant positive correlations were seen between CIP-3GC $(r=0.8921 ; p<0.001)$, CIP-SXT $(r=0.8007$; $p=0.005)$, and 3GC-SXT $(r=0.7373 ; p=0.015)$ (Figure 10, Table 10). 
Table 11. Correlation matrix of resistance data for inpatient Acinetobacter spp. UTI isolates among six indicator antibiotics (2008-2017).

\begin{tabular}{|c|c|c|c|c|c|c|c|}
\hline & CIP & IMI & MER & GEN & AMI & SXT & \\
\hline CIP & $X$ & 0.031 & 0.4486 & 0.022 & 0.5698 & 0.006 & \multirow{6}{*}{$\begin{array}{l}\text { Statistical } \\
\text { signifi- } \\
\text { cance } \\
(p=)\end{array}$} \\
\hline IMI & -0.6785 & $X$ & 0.3804 & 0.021 & 0.083 & 0.002 & \\
\hline MER & -0.2711 & 0.3119 & $X$ & 0.9967 & 0.8073 & 0.8697 & \\
\hline GEN & 0.7096 & -0.7102 & -0.001 & $X$ & 0.1456 & 0.007 & \\
\hline AMI & 0.2051 & -0.5735 & -0.0888 & 0.4952 & $X$ & 0.1561 & \\
\hline SXT & 0.7970 & -0.8416 & 0.0598 & 0.7857 & 0.4843 & $X$ & \\
\hline
\end{tabular}

Green cells denote Pearson-correlation coefficients representing strong $(0.5<|\mathrm{r}|<0.85)$ and statistically significant $(p<0.05)$ correlations; blue cells denote Pearson-correlation coefficients representing strong $(0.5<|\mathrm{r}|<0.85)$, but statistically non-significant $(p \geq 0.05)$ correlations; white cells denote Pearson-correlation coefficients representing moderate or weak $(|\mathrm{r}|<0.5)$, statistically non-significant $(p \geq 0.05)$ correlations. Legend: CIP: ciprofloxacin; IMI: imipenem; MER: meropenem; GEN: gentamicin; AMI: amikacin; SXT: trimethoprim-sulfamethoxazole.

Table 12. Correlation matrix of resistance data for inpatient Pseudomonas spp. UTI isolates among seven indicator antibiotics (2008-2017).

\begin{tabular}{|c|c|c|c|c|c|c|c|c|}
\hline & CIP & IMI & MER & CEFT & CEFE & GEN & AMI & \\
\hline CIP & $X$ & 0.7249 & 0.6326 & 0.7215 & 0.4219 & 0.091 & 0.5413 & \multirow{7}{*}{$\begin{array}{l}\text { Statistical } \\
\text { signifi- } \\
\text { cance } \\
(p=)\end{array}$} \\
\hline IMI & 0.1278 & $X$ & 0.9402 & 0.037 & 0.7006 & 0.5551 & 0.1633 & \\
\hline MER & -0.1731 & 0.027 & $X$ & 0.9351 & 0.8871 & 0.5665 & 0.3523 & \\
\hline CEFT & -0.1295 & -0.6612 & 0.0297 & $X$ & 0.072 & 0.3534 & 0.1695 & \\
\hline CEFE & 0.2867 & -0.1396 & 0.0517 & 0.5910 & $X$ & 0.004 & 0.048 & \\
\hline GEN & 0.5614 & -0.2128 & -0.2068 & 0.3289 & 0.8204 & $X$ & 0.002 & \\
\hline$\overline{\text { AMI }}$ & 0.2201 & -0.4771 & -0.3296 & 0.4709 & 0.6372 & 0.8565 & $X$ & \\
\hline
\end{tabular}

Orange cells denote Pearson-correlation coefficients representing very strong $(|r| \geq 0.85)$ and statistically significant $(p<0.05)$ correlations; green cells denote Pearson-correlation coefficients representing strong $(0.5<|\mathrm{r}|<0.85)$ and statistically significant $(p<0.05)$ correlations; blue cells denote Pearson-correlation coefficients representing strong $(0.5<|\mathrm{r}|<0.85)$, but statistically non-significant $(p \geq 0.05)$ correlations; white cells denote Pearsoncorrelation coefficients representing moderate or weak $(|\mathrm{r}|<0.5)$, statistically non-significant $(p \geq 0.05)$ correlations. Legend: CIP: ciprofloxacin; IMI: imipenem; MER: meropenem; CEFT: ceftazidime; CEFE: cefepime; GEN: gentamicin; AMI: amikacin.

In Acinetobacter spp., most notably, we observed strong and significant negative correlations between IMI-CIP $(\mathrm{r}=-0.6785 ; p=0.031)$, IMI-GEN $(\mathrm{r}=-0.7102 ; p=0.021)$, and IMI-SXT resistance $(\mathrm{r}=-0.8416 ; p=0.002)$, whereas positive correlations were noted for CIP-GEN $(r=0.7096 ; p=0.022)$ and CIP-SXT resistance $(r=0.7970 ; p=0.006)$, respectively (Figure 11, Table 11).

Lastly, in Pseudomonas spp., notable positive correlations were identified for GENCEFE $(r=0.8024 ; p=0.004$ and GEN-AMI $(r=0.8565 ; p=0.002)$, whereas negative correlation was seen between CEFT and IMI resistance $(\mathrm{r}=-0.6612 ; p=0.037)$ (Figure 12, Table 12).

\subsection{Discussion}

Antimicrobial resistance (AMR) is one of the most worrisome threats humanity has to face in the 21st century, which has been recognized by many government leaders and trans-national organizations [42]. Due to the extensive resistance in many of the Gramnegative bacteria, these pathogens are considered as a priority for R\&D and antimicrobial drug discovery platforms, to facilitate the development of new antibiotics [43]. In addition, continuous monitoring of resistance rates in a given institution or geographical region is another necessity to successfully address AMR globally [44]. Bacteria may present with the MDR/XDR phenotype through the contribution of a variety of mechanisms, including both intrinsic and acquired resistance [45]. The detailed knowledge of intrinsic resistance mechanisms in Gram-negative bacteria is critical for clinicians and infectious disease specialists, as they need to be considered even in cases of otherwise susceptible 
isolates. A summary of intrinsic resistance mechanisms seen in relevant Gram-negative bacteria is presented in Table 13.

Table 13. Summary of intrinsic resistance exhibited by Gram-negative bacteria included in this study (adapted from $[25,45,46])$.

\begin{tabular}{|c|c|}
\hline $\begin{array}{l}\text { All Gram-negative } \\
\text { bacteria }\end{array}$ & $\begin{array}{l}\text { glycopeptides (e.g., vancomycin), lincosamides (e.g., clindamycin), oxazolidinones (linezolid, tedizolid), } \\
\text { streptogramins (quinpristin-dalfopristin), macrolides (e.g., azithromycin), daptomycin, tetracycline }\end{array}$ \\
\hline Escherichia coli & - \\
\hline Klebsiella spp. & ampicillin \\
\hline $\begin{array}{l}\text { Citrobacter- } \\
\text { Enterobacter-Serratia } \\
\text { group }\end{array}$ & $\begin{array}{l}\text { CES: aminopenicillins, aminopenicillin/ } \beta \text {-lactamase-inhibitor combinations, I-II. generation } \\
\text { cephalosporins; Serratia spp.: nitrofurantoin, doxycycline, polymyxin B, colistin, aminoglycosides (except: } \\
\text { streptomycin, amikacin) }\end{array}$ \\
\hline $\begin{array}{l}\text { Proteus-Providencia- } \\
\text { Morganella } \\
\text { group }\end{array}$ & $\begin{array}{l}\text { resistance: aminopenicillins, aminopenicillin/ } \beta \text {-lactamase-inhibitor combinations, I-II. generation } \\
\text { cephalosporins (except for: P. mirabilis), nitrofurantoin, doxycycline, polymyxin B, colistin; reduced } \\
\text { susceptibility: imipenem }\end{array}$ \\
\hline Acinetobacter spp. & $\begin{array}{l}\text { aminopenicillins, aminopenicillin/ } \beta \text {-lactamase-inhibitor combinations, I-III. generation cephalosporins, } \\
\text { ertapenem, aztreonam, nitrofurantoin, doxycycline, fosfomycin }\end{array}$ \\
\hline Pseudomonas spp. & $\begin{array}{l}\text { aminopenicillins, aminopenicillin/ } \beta \text {-lactamase-inhibitor combinations, I-II. generation cephalosporins, } \\
\text { orally administered III. generation cephalosporins, ertapenem, trimethoprim-sulfamethoxazole, rifampin, } \\
\text { nitrofurantoin, doxycycline, tigecycline }\end{array}$ \\
\hline
\end{tabular}

Molecular tests and sequencing-based platforms are now considered as the goldstandard for AMR surveillance, providing detailed information on what kind of resistancedeterminants may be found in a given bacterial isolate, as well as the type of genetic information (chromosomal or MGE), which influences the possibility of rapid dissemination, especially in a nosocomial environment $[30,47]$. However, these technologies are not yet available to most routine clinical laboratories with high turnovers of clinical material. Moreover, the identification of a resistance gene alone does not predict the relationship between genotype and phenotype (i.e., the expression level of the gene), often leading to discrepancies [48]. For this reason, the standardized collection of phenotypic resistance data, and the introduction of Big Data analytic methods into AMR surveillance may be a viable alternative for molecular surveillance (and to maintain "regional" antibiograms), especially in low-resource settings [49]. Multivariate analyses of large datasets involving bacteria (e.g., resistance rates, expression of virulence factors, biofilm-formation), such as PCA and correlation matrices, have been performed [39]. For example, Amsalu et al. analyzed phenotypic data and sequencing in $n=147$ P. aeruginosa to assess the correlation between the resistance to biocide-resistance, and their results suggested that biocide resistance showed a significant positive correlation between biocide resistance and phenotypic resistance to fluoroquinolones, cephalosporins, and aminoglycosides [31]. Zhang et al. identified significant positive correlation among resistance and the presence of virulence genes associated with the extra-intestinal pathogenic (ExPEC) pathotype of E. coli isolated from healthy ducks [50]. Zhang et al. analyzed phenotypic and genotypic susceptibility data of foodborne pathogens from the NCBI Pathogen Detection Isolates Browser (NPDIB) database, corresponding to six US states, using PCA and hierarchical clusters [40]. They found that isolates from states in geographic proximity (Pennsylvania, New York, and Maryland) shared more similar resistance genes, and overall, the following ten genes were the most common contributors to the MDR phenotype: aadA; aph(3"); $a p h\left(3^{\prime \prime}\right)-I b$; aph(6)-I; aph (6)-Id; bla; blaCMY; tet; tet(A); and sul2 [40]. Li et al. utilized the same NPDIB database to assess phenotypic and genotypic susceptibility data from six different countries (Australia, Brazil, China, South Africa, the UK, and the US), and they have shown that geographical proximity was an important factor in identifying common resistance genes, and

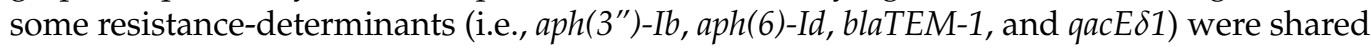
among all six countries. The authors have proposed, based on these historical resistance 
data, potential avenues for the spreading of antimicrobial resistance genes [51]. Mandal et al. studied the correlation between the multiple antibiotic resistance (MAR) phenotype and heavy metal $\left(\mathrm{Hg}^{2+}, \mathrm{Cd}^{2+}, \mathrm{Cr}^{2+}\right.$, and $\left.\mathrm{Cu}^{2+}\right)$ resistance in E. coli and non-fermenting Gram-negative bacteria isolated from sewage wastewater, and found significant correlation between MAR indices and heavy metal tolerance [52].

UTIs-especially in primary care settings-are treated empirically in most cases, due to the predictable range of pathogens implicated in these infections. Traditionally, NIT, FOS, NIT, and pivmecillinam are recommended for uncomplicated UTIs, whereas for more severe cases, fluoroquinolones and II-III. generation cephalosporins are often prescribed [53]. However, with the increase in MDR rates, these abovementioned drugs have decreased effectiveness, and the inappropriate empiric therapy of these infections leads to selection pressure, an additional burden for the patient and the healthcare system $[25,54]$. Though from an antimicrobial stewardship point of view, the management of UTIs may be considered as "low hanging fruit", with the mortality rate of these infections being much lower compared to invasive infections (e.g., sepsis, pneumonia) caused by the same bacteria, due to their high incidence and large patient population affected, these infections still have plenty of room for significant interventions to be made in the prudent and thoughtful utilization of antibiotics [55]. As a part of our secondary study, PCA and correlation analyses were performed, corresponding to resistance data of $>30,000$ UTIs, representing taxonomically-diverse Gram-negative pathogens, spanning over a 10-year long surveillance period from our tertiary-care hospital in Hungary. Our aim was to establish the co-occurrence of phenotypic resistance to indicator antibiotics in these isolates, to potentially uncover associations that were previously unaddressed, and to identify which resistances are the most relevant contributors to the development of the MDR phenotype (i.e., resistance to one agent in at least three different antibiotic groups [56]) in our setting. This information may direct therapeutic decisions to avoid the extensive use of some antimicrobials, decreasing unwanted selection pressure by these drugs.

This present study is a continuation of a comprehensive characterization of 10-years' worth of UTI resistance data in Southern Hungary [33]. Our data analyses have revealed some strong associations and co-occurrences of phenotypic resistance: in outpatient $E$. coli isolates, CIP resistance was a principal factor, commonly associated with GEN and SXT resistance, whereas in inpatient isolates, GEN resistance was the most common denominator, strongly associated with CIP, SXT, and resistance of 3GCs. In inpatient and outpatient E. coli alike, a strong negative correlation was seen between FOS and NIT resistance, both being commonly-used antimicrobials in the treatment of uncomplicated UTIs [54]. Interestingly, a slightly different picture was observed in outpatient and inpatient Klebsiella spp.: though in both cases, cohesion was seen in CIP resistance, in outpatient isolates, strong positive correlations were seen between CIP-GEN and CIP-3GC resistance, whereas for inpatient isolates, associations of CIP-SXT and CIP-FOS co-resistance were the most relevant. In the CES group isolates, strong correlations were seen among the resistance to most antimicrobials included: in the outpatient group, GEN resistance was the strongest common denominator, whereas in the inpatient group, 3GC resistance was the most relevant. Interestingly, in inpatient isolates, strong negative correlation was shown between FOS resistance and resistance to all other indicator antibiotics. In the PPM group isolates, $\mathrm{CIP}$ was the common axis of co-resistance, showing very strong, positive correlation with SXT in both groups (in addition to FOS and 3GC in the outpatient and inpatient group, respectively). In outpatient Acinetobacter spp. isolates, CIP showed strong and positive correlations with all other indicator antibiotics, in addition to IMI showing common cooccurrence with the two aminoglycoside drugs (GEN and AMI). In inpatient isolates, the significant co-occurrence of CIP with other antibiotics was less common, whereas SXT resistance showed strong positive associations with the members of a variety of antibiotic groups (i.e., IMI, GEN and CIP). In outpatient Pseudomonas spp., an interesting distinction was observed: strong positive correlation was seen between CEFE and other antibiotics (IMI, MER and GEN), whereas strong negative correlation was detected with CEFT and 
the same antimicrobials. In inpatient isolates, CEFE resistance showed strong positive co-occurrence with the two aminoglycoside drugs, whereas strong negative correlation was observed between CEFT-IMI. Co-occurrence of CIP resistance was not as relevant in Pseudomonas spp. as in other Gram-negative bacteria. Unsurprisingly, both in Pseudomonas spp. and Acinetobacter spp., strong and positive correlation was shown between the resistance to the two aminoglycoside drugs (GEN and AMI). The data presented herein may prove to be useful in complementing already existing antimicrobial stewardship interventions and hospital antibiograms. Based on our results, we have shown that-from our data-no overarching conclusions may be drawn for Gram-negative bacteria as a whole. As the presence of resistance to each individual indicator antibiotic had varying relevance in different taxonomic groups, this needs to be addressed accordingly. In addition, variations have occurred even between inpatient and outpatient isolates of the same taxonomic unit, which is most probably due to the different set of relevant antimicrobials prescribed in outpatient clinics and inpatient departments (with the opportunity to use antimicrobials in intravenous infusion form in the latter setting), as not all antibiotics are equally relevant in all patient groups (based on age or underlying conditions) [57].

Age and gender of the patients affected by UTIs has been suggested as an important epidemiological factor in forecasting resistance rates in urinary pathogens, i.e., with the increasing age of patients, resistance rates may also show an increasing trend [58]. Although we did not ascertain this correlation as a part of this study, our previous studies corresponding to the UTIs described in patients aged $\geq 65$ years of age, and in male patients (whom are affected by complicated UTIs, usually in advanced ages) have both shown higher rates of resistance to UTI-specific drugs (nitrofurantoin, fosfomycin), 3GCs, fluoroquinolones, and a higher prevalence of MDR isolates overall, compared to the general population [59,60]. The burden of UTIs in healthcare-associated infections has been highlighted by a nosocomial surveillance study in a 1600 -bed hospital in Eastern Hungary, where $21.1 \%$ of such infections were UTIs during the study period (2004-2006) [61]. In addition, a study by Bánhidy et al. reported that $5.7 \%$ of mothers were affected by UTIs during pregnancy, and these pregnancies had a higher proportion of preterm births (10.4\% vs. 9.1\%) [62]. The study of Szász et al. included the analysis of uropathogens from seven inpatient clinics of the Semmelweis University (Budapest) between the years 2006 and 2008, and the species distribution reported was similar to our study (Gram-negative bacteria in the majority, E. coli: $34-54 \%$, Klebsiella spp.: 3-11\%, Pseudomonas spp.: 3-9\%, the PPM group: 3.5-8\%, and Enterobacter spp.: 2-6\%), however, resistance rates reported were considerably lower in their paper [63]. The study of Illesy et al. described the prevalence of infections in kidney transplant recipients between 2010 and 2015 at the Faculty of Medicine, University of Debrecen: $69.7 \%$ of patients developed an infection, $79.3 \%(n=88)$ of the infections were UTIs (caused by E. coli, Enterococcus faecalis and Klebsiella spp.), and 19.8\% of the infections were MDR [64]. Finally, the recently-published microbial surveillance study of Magyar et al. described the UTI epidemiology at the urology department of the Jahn Ferenc South Pest Teaching Hospital (Budapest) between 2004 and 2015. E. coli and E. faecalis were shown to be the most common uropathogens, however, a slow but noticeable growth in the rates of P. mirabilis and P. aeruginosa was also seen throughout the study. Similar to our results, this study also highlighted the retained effectiveness of carbapenems and polymyxin $B$ for Enterobacterales, and high levels of fluoroquinolone resistance in most bacteria. In contrast to our study, they have shown very low resistance rates to fosfomycin $(0-15 \%)$ and nitrofurantoin $(<2 \%)$ in E. coli, and high resistance rates to 3GCs in Klebsiella spp. (owing to the high prevalence of ESBL-producers) [65].

It must be addressed that the rates of developing resistance (either through spontaneous mutations or through acquiring MGEs via horizontal gene transfer) may be slightly different between distinct antibiotic families [66]: for example, there are chromosomallyencoded $\beta$-lactamases in Enterobacterales and ESBLs, and now carbapenemases are located on MGEs (plasmids, integrons, or transposons), and are epidemiologically much more relevant, due to the risk of their rapid dissemination. For aminoglycoside-inactivating 
enzymes, chromosomal carriage and MGEs are equally important, whereas for highlevel phenotypic fluoroquinolone-resistance to occur, often the presence of more than resistance determinants (chromosomal and plasmid-mediated resistance, affecting DNA gyrase and topoisomerase IV, coupled with alterations in outer membrane proteins or overexpression of efflux pumps) is needed $[67,68]$. On the other hand, colistin-resistance and plasmid-borne colistin resistance (encoded by the mor genes) were first described in 2015 in E. coli and K. pneumoniae [69]. It has been extensively described-both in Hungary and in other countries-that acquiring plasmid-borne fluoroquinolone resistance is a critical step towards the development of MDR in both Enterobacterales positive or negative for ESBL-production (as also demonstrated by our data analysis) [70]. Subsequently, these isolates will present with chromosomal or plasmid-mediated aminoglycosideresistance and resistance to other ancillary antibiotics (such as nitrofurantoin, fosfomycin, trimethoprim-sulfamethoxazole, and next-generation tetracycline-derviatives) [71]. In the end, the process ends with infections that may only be treated by carbapenems, novel $\beta$-lactam/ $\beta$-lactamase-inhibitors, and colistin [72]. Tracing the steps towards the development of the MDR phenotype in non-fermenters (Acinetobacter spp., Pseudomonas spp.) is not as straightforward. Though resistance against fluoroquinolones and aminoglycosides may undoubtedly occur in these pathogens through similar mechanisms seen in Enterobacterales, the mechanisms and contribution of resistance to $\beta$-lactam antibiotics in non-fermenters is more diverse, with downregulation or absence of the OprD porins, efflux pump-overexpression, and penicillin-binding protein (PBP) modifications also having pronounced roles, in addition to $\beta$-lactamases [73-75]. Moreover, the presence of genotypic resistance mechanisms in these bacteria may affect the in vitro susceptibility of individual antibiotics - even in the same family of antibiotics—differently (e.g., often resulting in isolates non-susceptible to meropenem, but not imipenem) [76,77]. Our study highlights the importance of accumulating large datasets (either phenotypic or genotypic in nature) originating from microbiological isolates, and taking advantage of them in secondary data analyses for the purposes of basic science or clinical practice [78].

\section{Conclusions}

As the threat of AMR is reaching critical levels worldwide, the importance of timely surveillance regarding resistance rates-both for epidemiological purposes and to aid the selection of empiric therapy for clinicians-must be one of the key priorities of relevant stakeholders. This data collection may range from the simple collection of phenotypic resistance rates (to create a hospital antibiogram) to the extensive utilization of sequencing methods to monitor dominant MDR clones and genotypic resistance-determinants in the region, depending on the availability of the methods and funding. Though many microbiology laboratories (often serving large geographical regions) simply do not have the resources to maintain advanced sequencing platforms, they may possess considerably-sized longitudinal resistance data, which could provide valuable insights for AMR surveillance on a local and a national level. Our paper-detailing the co-occurrence and correlation of phenotypic resistance in Gram-negative UTIs for 10 years-may be considered a case study for the feasibility of standardized phenotypic resistance data collection and the use of data mining (considering resistance data as "Big Data") to be a cost-friendly alternative for molecular surveillance in resource-scarce settings. The syndrome-specific analyses of large resistance datasets (spanning over long periods of time) may show the decreasing relevance of some antibiotic groups in the treatment of the given bacterial infection, in addition to showing the tendency of resistances to major antibiotic groups presenting in parallel, acting as a "compass" to avoid therapeutic failure at the bedside.

Author Contributions: M.G. conceived and designed the study, performed data collection and statistical analysis, wrote and revised the full paper. Z.B. performed statistical analysis and prepared the figures. K.B. provided the original data, wrote and revised the full paper. All authors have read and agreed to the published version of the manuscript. 
Funding: M.G. and Z.B. were supported by the János Bolyai Research Scholarship (M.G.: BO/00144/ 20/5; Z.B.: BO/00298/21/8) of the Hungarian Academy of Sciences. The research was supported by the UNKP-21-5 New National Excellence Program (M.G.: ÚNKP-21-5-540-SZTE, Z.B.: ÚNKP-21-5581-SZTE) of the Ministry for Innovation and Technology from the source of the National Research. Development and Innovation Fund. The contribution of Z.B. was also supported by the NKFIH K 124796 grant. M.G. would also like to acknowledge the support of ESCMID's “30 under 30" Award.

Institutional Review Board Statement: Our research complies with the Declaration of Helsinki (1964) and its amendments. As data anonymity was maintained throughout the data collection and assessment periods, the present study was not subject to ethics review. Informed consent was not required due to the retrospective nature of the study.

Informed Consent Statement: Not applicable.

Data Availability Statement: The primary data used during the analyses of this study may be found in the publication of Gajdács et al. (https://www.mdpi.com/2075-1729/10/2/16, accessed on 8 August 2021).

Acknowledgments: Not applicable.

Conflicts of Interest: The authors declare no conflict of interest to disclose, monetary or otherwise.

\section{References}

1. Lobanovska, M.; Pilla, G. Penicillin's Discovery and Antibiotic Resistance: Lessons for the Future? Yale J. Biol. Med. 2017, 90, 135-145.

2. Gajdács, M.; Urbán, E.; Stájer, A.; Baráth, Z. Antimicrobial Resistance in the Context of the Sustainable Development Goals: A Brief Review. Eur. J. Investig. Health Psychol. 2021, 11, 71-82. [CrossRef]

3. Levy, S.B.; Marshall, B. Antibacterial resistance worldwide: Causes, challenges and responses. Nat. Med. 2004, 10, S122-S129. [CrossRef]

4. $\quad$ Olesen, S.W.; Barnett, M.L.; MacFadden, D.R.; Brownstein, J.S.; Hernández-Díaz, S.; Lipsitch, M.; Grad, Y.H. The distribution of antibiotic use and its association with antibiotic resistance. eLife 2018, 7, e39435. [CrossRef]

5. Medina, E.; Pieper, D.H. Tackling Threats and Future Problems of Multidrug-Resistant Bacteria. Curr. Top. Microbiol. Immunol. 2016, 398, 3-33.

6. Aslam, A.; Gajdács, M.; Zin, C.S.; Rahman, N.S.A.; Ahmed, S.I.; Zafar, M.Z.; Jamshed, S. Evidence of the Practice of SelfMedication with Antibiotics among the Lay Public in Low- and Middle-Income Countries: A Scoping Review. Antibiotics 2020, 9, 597. [CrossRef] [PubMed]

7. ECDC/EMEA Joint Technical Report (2009). The Bacterial Challenge: Time to React. Available online: http://ecdc.europa.eu/ en/publications/Publications/0909_TER_The_Bacterial_Challenge_Time_to_React.pdf. (accessed on 5 August 2021).

8. CDC Antibiotic/Antimicrobial Resistance (AR/AMR). Available online: https://www.cdc.gov/drugresistance/biggest_threats. html (accessed on 5 August 2021).

9. WHO. WHO Publishes List of Bacteria for Which New Antibiotics Are Urgently Needed. Available online: https://www. who.int/news/item/27-02-2017-who-publishes-list-of-bacteria-for-which-new-antibiotics-are-urgently-needed (accessed on 5 August 2021).

10. O'Neill, J. Antimicrobial Resistance: Tackling a Crisis for the Health and Wealth of Nations. Available online: https:// wellcomecollection.org/works/rdpck35v/items (accessed on 5 August 2021).

11. Santajit, S.; Indrawattana, N. Mechanisms of Antimicrobial Resistance in ESKAPE Pathogens. Biomed. Res. Int. 2016, 2016, 2475067. [CrossRef] [PubMed]

12. Van Hoek, A.H.A.M.; Mevius, D.; Guerra, B.; Mullany, P.; Roberts, A.P.; Aarts, H.M.J. Acquired Antibiotic Resistance Genes: An Overview. Front. Microbiol. 2011, 2, e203. [CrossRef]

13. Boto, L. Horizontal gene transfer in evolution: Facts and challenges. Proc. R. Soc. B 2010, 277, 819-827. [CrossRef]

14. Li, Q.; Chang, W.; Zhang, H.; Hu, D.; Wang, X. The Role of Plasmids in the Multiple Antibiotic Resistance Transfer in ESBLsProducing Escherichia coli Isolated from Wastewater Treatment Plants. Front. Microbiol. 2019, 10, e633. [CrossRef] [PubMed]

15. Gajdács, M. The Concept of an Ideal Antibiotic: Implications for Drug Design. Molecules 2019, 24, 892. [CrossRef]

16. Melter, O.; Radojevic, M. Small colony variants of Staphylococcus aureus-Review. Folia Microbiol. 2010, 55, 548-558. [CrossRef]

17. Cui, X.; Zhang, H.; Du, H. Carbapenemases in Enterobacteriaceae: Detection and Antimicrobial Therapy. Front. Microbiol. 2019, 10, e1823. [CrossRef]

18. Rapoport, M.; Faccone, D.; Pasteran, F.; Ceriana, P.; Albornoz, E.; Petroni, A.; Corso, A.; The MCR Group. First Description of mcr-1-Mediated Colistin Resistance in Human Infections Caused by Escherichia coli in Latin America. Antimicrob. Agents Chemother. 2016, 60, 4412-4413. [CrossRef]

19. Foxman, B. Urinary tract infection syndromes: Occurrence, recurrence, bacteriology, risk factors and disease burden. Infect. Dis. Clin. N. Am. 2014, 28, 1-13. [CrossRef] [PubMed] 
20. Johansen, T.E.B.; Botto, H.; Cek, M.; Grabe, M.; Tenke, P.; Wagenlehner, M.E.; Naber, K.G. Critical review of current definitions of urinary tract infections and proposal of an EAU/ESIU classification system. Int. J. Antimicrob. Agents 2011, 38, 64-70. [CrossRef] [PubMed]

21. Klein, R.D.; Hultgren, S.J. Urinary tract infections: Microbial pathogenesis, host-pathogen interactions and new treatment strategies. Nat. Rev. Microbiol. 2020, 18, 211-226. [CrossRef] [PubMed]

22. Simmering, J.E.; Tang, F.; Cavanaugh, J.E.; Polgreen, L.A.; Polgreen, P.M. The increase in hospitalizations for urinary tract infections and the associated cost in the United States, 1998-2011. Open Forum Infect. Dis. 2017, 4, ofw281. [CrossRef]

23. Petca, R.C.; Negoita, S.; Mares, C.; Petca, A.; Popescu, R.I.; Chibelean, C.B. Heterogeneity of Antibiotics Multidrug-Resistance Profile of Uropathogens in Romanian Population. Antibiotics 2021, 10, 523. [CrossRef] [PubMed]

24. Petca, R.C.; Mares, C.; Petca, A.; Negoita, S.; Popescu, R.I.; Bot, M.; Barabás, E.; Chibelean, C.B. Spectrum and Antibiotic Resistance of Uropathogens in Romanian Females. Antibiotics 2020, 9, 472. [CrossRef]

25. Behzadi, P.; Urbán, E.; Matuz, M.; Benkő, R.; Gajdács, M. The Role of Gram-Negative Bacteria in Urinary Tract Infections: Current Concepts and Therapeutic Options. Adv. Exp. Med. Biol. 2021, 1323, 35-69.

26. Hrbacek, J.; Cermak, P.; Zachoval, R. Current antibiotic resistance patterns of rare uropathogens: Survey from Central European Urology Department 2011-2019. BMC Urol. 2021, 21, e61. [CrossRef]

27. Fuhrmeister, A.S.; Jones, R.N. The Importance of Antimicrobial Resistance Monitoring Worldwide and the Origins of SENTRY Antimicrobial Surveillance Program. Open Forum Infect. Dis. 2019, 6, S1-S4. [CrossRef]

28. Majumder, M.A.A.; Rahman, S.; Cohall, D.; Bharatha, A.; Singh, K.; Haque, M.; Hilarie, M.G.S. Antimicrobial Stewardship: Fighting Antimicrobial Resistance and Protecting Global Public Health. Infect. Drug. Res. 2020, 13, 4713-4738. [CrossRef] [PubMed]

29. Vu, T.L.H.; Vu, Q.D.; Hoang, B.L.; Nguyen, T.C.T.; Ta, T.D.N.; Nadjm, B.; van Doorn, H.R. Factors influencing choices of empirical antibiotic treatment for bacterial infections in a scenario-based survey in Vietnam. JAC-AMR 2020, 2, dlaa087. [CrossRef] [PubMed]

30. Riedel, S.; Halls, J.; Dutta, S.; Toraskar, N.; Lemon, J.; Carter, K.; Sinclair, W.; Lopansri, B.K.; Styer, A.M.; Wolk, D.M.; et al. Clinical evaluation of the acuitas AMR gene panel for the rapid detection of bacteria and genotypic antibiotic resistance determinants Diagn. Microbiol. Infect. Dis. 2021, 100, e115383. [CrossRef] [PubMed]

31. Amsalu, A.; Sapula, S.A.; Lopes, M.D.B.; Hart, B.J.; Nguyen, A.H.; Drigo, B.; Turnidge, J.; Venter, H. Efflux Pump-Driven Antibiotic and Biocide Cross-Resistance in Pseudomonas aeruginosa Isolated from Different Ecological Niches: A Case Study in the Development of Multidrug Resistance in Environmental Hotspots. Microorganisms 2020, 8, 1467. [CrossRef] [PubMed]

32. Van Katwyk, S.R.; Hoffman, S.J.; Mendelson, M.; Taljaard, M.; Grimshaw, J.M. Strengthening the science of addressing antimicrobial resistance: A framework for planning, conducting and disseminating antimicrobial resistance intervention research. Health Res. Policy Syst. 2020, 18, e60. [CrossRef]

33. Gajdács, M.; Bátori, Z.; Ábrók, M.; Lázár, A.; Burián, K. Characterization of Resistance in Gram-Negative Urinary Isolates Using Existing and Novel Indicators of Clinical Relevance: A 10-Year Data Analysis. Life 2020, 10, 16. [CrossRef]

34. Hospital Bed Count and Patient Turnover Report 2017. National Health Insurance Fund of Hungary. Available online: http:/ / www.neak.gov.hu/felso_menu/szakmai_oldalak/publikus_forgalmi_adatok/gyogyito_megelozo_forgalmi_adat/ fekvobeteg_szakellatas/korhazi_agyszam.html (accessed on 5 August 2021).

35. Hungarian Ministry of Health. Guidelines for the Microbiological Diagnostics of Uncomplicated Urinary Tract Infections. Available online: https:/ / old-kollegium.aeek.hu/conf/upload/oldiranyelvek/ORV-MIK_A\%20klasszikus\%20h\%c3\%bagy\% c3\%bati\%20infekci\%c3\%b3k\%20mikrobiol\%c3\%b3giai\%20diagnosztik\%c3\%a1j\%c3\%a1r\%c3\%b31_mod0_v0.pdf (accessed on 5 August 2021).

36. Gajdács, M.; Ábrók, M.; Lázár, A.; Burián, K. Comparative Epidemiology and Resistance Trends of Common Urinary Pathogens in a Tertiary-Care Hospital: A 10-Year Surveillance Study. Medicina 2019, 55, 356. [CrossRef]

37. EUCAST Clinical Breakpoints and Dosing. Available online: https://www.eucast.org/clinical_breakpoints/ (accessed on 5 August 2021).

38. EUCAST Guideline for the Detection of Resistance Mechanisms and Specific Resistances of Clinical and/or Epidemiological Importance. Available online: https://www.eucast.org/fileadmin/src/media/PDFs/EUCAST_files/Resistance_mechanisms/ EUCAST_detection_of_resistance_mechanisms_170711.pdf (accessed on 5 August 2021).

39. Sadat, A.; El-Sherbiny, H.; Zakaria, A.; Ramadan, H.; Awad, A. Prevalence, antibiogram and virulence characterization of Vibrio isolates from fish and shellfish in Egypt: A possible zoonotic hazard to humans. J. Appl. Microbiol. 2021, 131, 485-498. [CrossRef]

40. Zhang, N.; Liu, E.; Tang, A.; Ye, M.C.; Wang, M.C.; Wang, K.; Jia, Q.; Huang, Z. Data-Driven Analysis of Antimicrobial Resistance in Foodborne Pathogens from Six States within the US. Int. J. Environ. Res. Public Health 2019, 16, 1811. [CrossRef]

41. Mukaka, M.M. A guide to appropriate use of Correlation coefficient in medical research. Malawi Med. J. 2012, $24,69-71$.

42. Ali, J.; Rafiq, Q.A.; Ratcliffe, E. Antimicrobial resistance mechanisms and potential synthetic treatments. Future Sci. 2018, 4, FSO290. [CrossRef] [PubMed]

43. Karaiskos, I.; Galani, I.; Souli, M.; Giamarellou, H. Novel $\beta$-lactam- $\beta$-lactamase inhibitor combinations: Expectations for the treatment of carbapenem-resistant Gram-negative pathogens. Expert Opin. Drug Metab. Toxicol. 2019, 15, 133-149. [CrossRef] [PubMed] 
44. Bandyopadhyay, S.; Bhattacharyya, D.; Samanta, I.; Banerjee, J.; Habib, M.; Dutta, T.K.; Dutt, T. Characterization of MultidrugResistant Biofilm-Producing Escherichia coli and Klebsiella pneumoniae in Healthy Cattle and Cattle with Diarrhea. Microb. Drug. Res. 2021. [CrossRef] [PubMed]

45. Juraschek, K.; Borowiak, M.; Tausch, S.H.; Malorny, B.; Kasbohrer, A.; Otani, S.; Schwarz, S.; Meemken, D.; Deneke, C.; Hammerl, J.A. Outcome of Different Sequencing and Assembly Approacheson the Detection of Plasmids and Localization of Antimicrobial Resistance Genes in Commensal Escherichia coli. Microorganisms 2021, 9, 598. [CrossRef] [PubMed]

46. EUCAST Expert Rules and Intrinsic Resistance. Available online: https://www.eucast.org/fileadmin/src/media/PDFs/ EUCAST_files/Expert_Rules/Expert_rules_intrinsic_exceptional_V3.1.pdf (accessed on 28 September 2021).

47. Lucassen, R.; Rehberg, L.; Heyden, M.; Bockmühl, D. Strong correlation of total phenotypic resistance of samples from household environments and the prevalence of class 1 integrons suggests for the use of the relative prevalence of intI 1 as a screening tool for multi-resistance. PLoS ONE 2019, 14, e0218277. [CrossRef]

48. Mahfouz, N.; Ferreira, I.; Beisken, S.; van Haeseler, A.; Posch, A.E. Large-scale assessment of antimicrobial resistance marker databasescfor genetic phenotype prediction: A systematic review. J. Antimicrob. Chemother. 2020, 75, 3099-3108. [CrossRef]

49. Ryu, S.; Cowling, B.J.; Wu, P.; Olesen, S.; Fraser, C.; Sun, D.S.; Lipsitch, M.; Grad, Y.H. Case-based surveillance of antimicrobial resistance with full susceptibility profiles. JAC Antimicrob. Resist. 2019, 1, dlz070. [CrossRef] [PubMed]

50. Zhang, S.; Chen, S.; Rehman, M.U.; Yang, H.; Yang, Z.; Wang, M.; Jia, R.; Chen, S.; Liu, M.; Zhu, D.; et al. Distribution and association of antimicrobial resistance and virulence traits in Escherichia coli isolates from healthy waterfowls in Hainan, China Ecotoxicol. Environ. Safety 2021, 220, e112317. [CrossRef] [PubMed]

51. Li, K.; Zheng, J.; Deng, T.; Peng, J.; Daniel, D.; Jia, Q.; Huang, Z. An Analysis of Antimicrobial Resistance of Clinical Pathogens from Historical Samples for Six Countries. Processes 2019, 7, 964. [CrossRef]

52. Mandal, M.; Das, N.S.; Mandal, S. Principal component analysis exploring the association between antibiotic resistance and heavy metal tolerance of plasmid-bearing sewage wastewater bacteria of clinical relevance. Access Microbiol. 2020, 2. [CrossRef]

53. Flores-Mireles, A.L.; Walker, J.N.; Caparon, M.; Hultgren, S.J. Urinary tract infections: Epidemiology, mechanisms of infection and treatment options. Nat. Rev. Microbiol. 2015, 13, 269-284. [CrossRef]

54. Kasanga, M.; Mukosha, R.; Kasanga, M.; Siyanga, M.; Mudenda, S.; Solochi, B.B.; Chileshe, M.; Mwiikisa, M.J.; Gondwe, T.; Kantenga, T.; et al. Antimicrobial resistance patterns of bacterial pathogens: Their distribution in university teaching hospitals in Zambia. Future Med. 2021, 16, 811-824. [CrossRef]

55. Abbo, L.M.; Hooton, T.M. Antimicrobial Stewardship and Urinary Tract Infections. Antibiotics 2014, 3, 175-192. [CrossRef] [PubMed]

56. Magiorakos, A.P.; Srinivasan, A.; Carey, R.B.; Carmeli, Y.; Falagas, M.E.; Giske, C.G.; Paterson, D.L. Multidrug-resistant, extensively drug-resistant and pandrug-resistant bacteria: An international expert proposal for interim standard definitions for acquired resistance. Clin. Microbiol. Infect. 2012, 18, 268-281. [CrossRef]

57. Artero, A.; Esparcia, A.; Eiros, J.M.; Madrazo, M.; Alberola, J.; Nogueria, J.M. Effect of Bacteremia in Elderly Patients With Urinary Tract Infection. Am. J. Med. Sci. 2016, 352, 267-271. [CrossRef]

58. Rowe, T.A.; Juthani-Mehta, M. Urinary tract infection in older adults. Aging Health 2013, 9, 519-528. [CrossRef] [PubMed]

59. Gajdács, M.; Ábrók, M.; Lázár, A.; Burián, K. Urinary Tract Infections in Elderly Patients: A 10-Year Study on Their Epidemiology and Antibiotic Resistance Based on the WHO Access, Watch, Reserve (AWaRe) Classification. Antibiotics 2021, 10, 1098. [CrossRef] [PubMed]

60. Gajdács, M.; Ábrók, M.; Lázár, A.; Burián, K. Epidemiology and antibiotics resistance profile of bacterial uropathogens in male patients: A 10-year retrospective study. Farmacia 2021, 69, 530-539. [CrossRef]

61. Orosi, P.; Farkas, A.; Berkes, I.; Salné, N.G.; Szentkereszty, Z.; Mályi, K.; Dán, A. Surveillance results of nosocomial infections of the ICU in Kenézy Hospital, based on two years data. Orv. Hetil. 2007, 148, 1469-1473. [CrossRef] [PubMed]

62. Bánhidy, F.; Acs, N.; Puhó, H.E.; Czeizel, A.E. Pregnancy complications and birth outcomes of pregnant women with urinary tract infections and related drug treatments. Scand. J. Infect. Dis. 2007, 39, 390-397. [CrossRef]

63. Szász, M.; Lehotkai, N.; Kristóf, K.; Szabó, D.; Nagy, K. Prevalence and antimicrobial resistance of uropathogens in different inpatient wards. Acta Microbiol. Immunol. Hung. 2009, 56, 375-387. [CrossRef]

64. Illesy, L.; Szabo-Pap, M.; Toth, F.; Zadori, G.; Zsom, L.; Asztalos, L.; Szabo, R.P.; Fedor, R.; Nemes, B. Bacterial Infections After Kidney Transplantation: A single-center experience. Transpl. Proc. 2016, 48, 2540-2543. [CrossRef]

65. Magyar, A.; Köves, B.; Nagy, K.; Dobák, A.; Arthanareeswaran, V.K.A.; Bálint, P.; Wagenlehner, F.; Tenke, P. Spectrum and antibiotic resistance of uropathogens between 2004 and 2015 in a tertiary care hospital in Hungary. J. Med. Microbiol. 2017, 66, 788-797. [CrossRef]

66. Porob, S.; Craddock, H.A.; Motro, Y.; Sagi, O.; Gdalevich, M.; Ezery, Z.; Davidovich, N.; Ronen, Z.; Moran-Gilad, J. Quantification and Characterization of Antimicrobial Resistance in Greywater Discharged to the Environment. Water 2020, 11, 1460. [CrossRef]

67. Machuca, J.; Briales, A.; Labrador, G.; Diaz-de-Alba, P.; López-Rojas, R.; Docobo-Pérez, F.; Martínez-Martínez, L.; Rodriguez-Bano, J.; Pachón, M.E.; Pascual, A.; et al. Interplay between plasmid-mediated and chromosomal-mediated fluoroquinolone resistance and bacterial fitness in Escherichia coli. J. Antimicrob. Chem. 2014, 69, 3203-3215. [CrossRef] [PubMed]

68. Galimand, M.; Couvalin, P.; Lambert, T. Plasmid-Mediated High-Level Resistance to Aminoglycosides in Enterobacteriaceae Due to 16S rRNA Methylation. Antimicrob. Agents Chemother. 2003, 47, 2565-2571. [CrossRef] [PubMed] 
69. Liu, Y.Y.; Wang, Y.; Walsh, T.R.; Yi, L.X.; Zhang, R.; Spencer, J.; Doi, Y.; Tian, G.; Dong, B.; Huang, X.; et al. Emergence of plasmid-mediated colistin resistance mechanism MCR-1 in animals and human beings in China: A microbiological and molecular biological study. Lancet Infect Dis. 2016, 16, 161-168. [CrossRef]

70. Szabó, O.; Gulyás, D.; Szabó, N.; Kristóf, K.; Kocsis, B.; Szabó, D. Plasmid-mediated quinolone resistance determinants in Enterobacteriaceae from urine clinical samples. Acta Microbiol. Immunol. Hung. 2018, 65, 255-265. [CrossRef] [PubMed]

71. Gulyás, D.; Kocsis, B.; Szabó, D. Plasmid copy number and qnr gene expression in selection of fluoroquinolone-resistant Escherichia coli. Acta Microbiol. Immunol. Hung. 2019, 66, 169-178. [CrossRef] [PubMed]

72. Knopp, M.; Babina, M.A.; Gudmundsdóttir, J.S.; Douglass, M.V.; Trent, M.S.; Andresson, D.I. A novel type of colistin resistance genes selected from random sequence space. PLoS Genet. 2021, 17, e1009227. [CrossRef]

73. Islam, S.; Akand, A.R.; Nova, T.T.; Lehmann, C.; Chisti, M.J. Sensitivity Patterns of Bacterial Pathogens Isolated from Blood Cultures of Under-Five Children with Pneumonia and Clinical Sepsis. Life 2021, 11, 450. [CrossRef] [PubMed]

74. Ferreria, M.; Sousa, C.F.; Gameiro, P. Fluoroquinolone Metalloantibiotics to Bypass Antimicrobial Resistance Mechanisms: Decreased Permeation through Porins. Membranes 2021, 11, 3. [CrossRef] [PubMed]

75. Bonomo, R.A.; Szabó, D. Mechanisms of multidrug resistance in Acinetobacter species and Pseudomonas aeruginosa. Clin. Infect. Dis. 2006, 43, S49-S56. [CrossRef] [PubMed]

76. Behzadi, P.; Baráth, Z.; Gajdács, M. It's Not Easy Being Green: A Narrative Review on the Microbiology, Virulence and Therapeutic Prospects of Multidrug-Resistant Pseudomonas aeruginosa. Antibiotics 2021, 10, 42. [CrossRef]

77. Kumar, A.B.; Thankappan, B.; Jayaraman, A.; Gupta, A. Evaluation of Antibiotic Tolerance in Pseudomonas aeruginosa for Aminoglycosides and Its Predicted Gene Regulations through In-Silico Transcriptomic Analysis. Microbiol. Res. 2021, 12, 630-645. [CrossRef]

78. Mhondoro, M.; Ndlovu, N.; Donewell, B.; Juru, T.; Tarafa, N.G.; Gerald, S.; Peter, N.; Mufuta, T. Trends in antimicrobial resistance of bacterial pathogens in Harare, Zimbabwe, 2012-2017: A secondary dataset analysis. BMC Infect. Dis. 2019, 19, e746. [CrossRef] 